\title{
Direct numerical simulation of the autoignition of a hydrogen plume in a turbulent coflow of hot air
}

\author{
Journal Article \\ Author(s): \\ Kerkemeier, S. G.; Markides, C. N.; Frouzakis, C. E.; Boulouchos, Konstantinos \\ Publication date: \\ 2013-04 \\ Permanent link: \\ https://doi.org/10.3929/ethz-b-000064749 \\ Rights / license: \\ In Copyright - Non-Commercial Use Permitted \\ Originally published in: \\ Journal of Fluid Mechanics 720, https://doi.org/10.1017/jfm.2013.22
}




\title{
Direct numerical simulation of the autoignition of a hydrogen plume in a turbulent coflow of hot air
}

\author{
S. G. Kerkemeier ${ }^{1}$, C. N. Markides ${ }^{2}$, C. E. Frouzakis ${ }^{1,} \dagger$ and K. Boulouchos ${ }^{1}$ \\ ${ }^{1}$ Aerothermochemistry and Combustion Systems Laboratory, Swiss Federal Institute of Technology, \\ Sonneggstrasse 3, CH-8092 Zurich, Switzerland \\ ${ }^{2}$ Department of Chemical Engineering, Imperial College London, South Kensington Campus, \\ London SW7 2AZ, UK
}

(Received 12 January 2012; revised 18 November 2012; accepted 6 January 2013; first published online 27 February 2013)

The autoignition of an axisymmetric nitrogen-diluted hydrogen plume in a turbulent coflowing stream of high-temperature air was investigated in a laboratory-scale set-up using three-dimensional numerical simulations with detailed chemistry and transport. The plume was formed by releasing the fuel from an injector with bulk velocity equal to that of the surrounding air coflow. In the 'random spots' regime, autoignition appeared randomly in space and time in the form of scattered localized spots from which post-ignition flamelets propagated outwards in the presence of strong advection. Autoignition spots were found to occur at a favourable mixture fraction close to the most reactive mixture fraction calculated a priori from considerations of homogeneous mixtures based on inert mixing of the fuel and oxidizer streams. The value of the favourable mixture fraction evolved in the domain subject to the effect of the scalar dissipation rate. The hydroperoxyl radical appeared as a precursor to the build-up of the radical pool and the ensuing thermal runaway at the autoignition spots. Subsequently, flamelets propagated in all directions with complex dynamics, without anchoring or forming a continuous flame sheet. These observations, as well as the frequency of and scatter in appearance of the spots, are in good agreement with experiments in a similar set-up. In agreement with experimental observations, an increase in turbulence intensity resulted in a downstream shift of autoignition. An attempt is made to understand the key processes that control the mean axial and radial locations of the spots, and are responsible for the observed scatter. The advection of the most reactive mixture through the domain, and hence the history of evolution of the developing radical pools were considered to this effect.

Key words: combustion, reacting flows, turbulent reacting flows

\section{Introduction}

Turbulent autoigniting flows pose a challenging fundamental problem due to their multiscale nature and the direct coupling between various physicochemical processes including the turbulent mixing bringing the fuel and oxidizer together, the subsequent complex pre-ignition chemistry between the reactants, and the fate of the heat and

$\dagger$ Email address for correspondence: frouzakis@lav.mavt.ethz.ch 
radicals produced from the chemical reactions leading to thermal runaway. At the same time there is a strong practical interest in novel low-emission concepts for power generation. In terms of enhanced performance and higher efficiency, the development of systems based on these concepts can be aided significantly by an improved understanding and ability to predict autoignition in the presence of finite fluctuations of velocity, temperature and composition. An extensive discussion of the progress in this field can be found in the review by Mastorakos (2009).

Direct numerical simulation (DNS) studies have provided insight into the processes that lead to autoignition in turbulent inhomogeneous flows, revealing thermal runaway at localized sites called 'autoignition kernels', followed by the explosive emergence of localized flamelets, which then propagate into their unburnt surroundings. Using high activation energy asymptotics, Liñán \& Crespo (1976) reported that autoignition in laminar conditions appeared first at locations that correspond to a specific value of fuel concentration. Similarly, the simulations of stagnant mixing layers in twodimensional isotropic decaying turbulence employing single-step Arrhenius chemistry (Mastorakos, Baritaud \& Poinsot 1997a) showed that autoignition starts at the 'most reactive' mixture fraction $\xi_{M R}$, a well-defined value of the mixture fraction $\xi$ where the reaction rate attains its highest value, which could be estimated a priori from homogeneous reactor calculations.

Mastorakos et al. (1997a) further observed that autoignition appeared at locations along the $\xi_{M R}$ isoline experiencing the lowest scalar dissipation rate,

$$
\chi=D(\nabla \xi)^{2}
$$

where $D=\lambda / \rho c_{p}$ is the local thermal diffusivity with $\rho, \lambda$ and $c_{p}$ the mixture density, conductivity and heat capacity, respectively. In the current study, the mixture fraction definition of Bilger, Stårner \& Kee (1990),

$$
\xi=\frac{0.5\left(Y_{H}-Y_{H, o x}\right) / W_{H}-\left(Y_{O}-Y_{O, o x}\right) / W_{O}}{0.5\left(Y_{H, f}-Y_{H, o x}\right) / W_{H}-\left(Y_{O, f}-Y_{O, o x}\right) / W_{O}}
$$

is adopted, where the subscripts ' $f$ ' and ' $o x$ ' refer to the values in the fuel and oxidizer stream, and $Y_{e}$ and $W_{e}$ are the elemental mass fraction and atomic weight of element $e$. The optimal conditions for autoignition in the sense of maximized reaction rates and minimal heat and radical losses are offered at locations along the $\xi_{M R}$ isosurface where the conditional scalar dissipation rate $\chi_{M R} \equiv \chi \mid \xi=\xi_{M R}$ is the lowest (Mastorakos et al. $1997 a)$; the notation $\chi \mid \xi=\xi_{M R}$ expresses the value of $\chi$ given that $\xi=\xi_{M R}$. This result highlighted that in turbulent autoignition the mean as well as the fluctuations of $\chi_{M R}$ play an important role. Ensuing numerical studies with detailed chemistry and transport in the presence of two-dimensional (Im, Chen \& Law 1998; Hilbert \& Thèvenin 2002; Sreedhara \& Lakshmisha 2002; Echekki \& Chen 2003; Viggiano \& Magi 2004) and three-dimensional turbulence (Sreedhara \& Lakshmisha 2002) have confirmed and extended the findings concerning the importance of $\xi_{M R}$ and $\chi_{M R}$ for turbulent autoignition.

Using different flow initializations, Mastorakos et al. (1997a) further reported that, although the partial premixing of the fuel with oxidizer or the increase of the thickness of the fuel-oxidizer interface (resulting in both cases to a decrease of $\chi_{M R}$ ) reduced the autoignition delay time $\tau_{i g n}$, the turbulent time scale $\tau_{t}$ did not affect $\tau_{i g n}$ beyond the statistical variation of the calculation. This outcome was in contrast to experimental observations available at the time, according to which enhanced mixing was seen as a means of promoting spray autoignition (reducing $\tau_{i g n}$ ) (Wong \& Steere 1982; 
Mizutani, Nakabe \& Chung 1990; Baritaud, Heinze \& Coz 1994). The limited effect of turbulence was attributed to the chemical induction time $\tau_{i g n}$ being similar to or smaller than the turbulent time scales, i.e. $\tau_{i g n} \leqslant \tau_{t}$, and it was predicted that for $\tau_{i g n} \gg \tau_{t}$, the significant variations developed in the history of the scalar dissipation rate at the autoignition location during the induction time will lead to a large spread in the emergence of autoignition.

By performing simulations over a wider range of conditions, Mastorakos, da Cruz, Baritaud \& Poinsot (1997b) demonstrated that enhanced turbulence (i.e. shorter $\tau_{t}$ ) resulted in a slight acceleration of autoignition and a small reduction of $\tau_{i g n}$. Of specific interest was the extension of the turbulent Reynolds number based on the initial turbulent kinetic energy $k$, the turbulent integral length scale $L_{t}$, and the kinematic viscosity $v$ from $R e_{t}=k^{1 / 2} L_{t} / v=60$ to 100 in Mastorakos et al. (1997a) to close to 450 . We note that for isotropic two-dimensional turbulence $k^{1 / 2}=u^{\prime}$, $L_{t}=k^{3 / 2} / \epsilon$ and $\tau_{t}=k / \epsilon$, where $u^{\prime}$ is the root mean square (r.m.s.) of the turbulent velocity fluctuations and $\epsilon$ is the dissipation rate of $k$.

The effect of two-dimensional turbulence on autoignition was studied further in DNS of a hydrogen-air mixing layer in homogeneous isotropic turbulence (Im et al. 1998) using a detailed chemical mechanism and an airstream temperature higher than the cross-over temperature where the chain branching and three-body termination reaction rates are equal. A non-monotonic effect of turbulence intensity on autoignition delay was observed, but the variation was quite small. Using a single-step irreversible reaction for segregated $n$-heptane packets in hot air, Sreedhara \& Lakshmisha (2000) found that increased initial $u^{\prime}$ of the isotropic, homogenous and decaying turbulent field delays autoignition in a two-dimensional periodic box, while in the threedimensional case and using a four-step kinetic scheme the autoignition delay time was found to either decrease when $\tau_{i g n} \gg \tau_{t}$ or remain unaffected when $\tau_{i g n} \leqslant \tau_{t}$.

Experiments in turbulent counterflowing jets of hydrogen and hot air (Blouch et al. 1998; Blouch \& Law 2003) demonstrated that a higher critical temperature was necessary for autoignition when the turbulence intensity in the heated air stream was increased, suggesting that increased turbulence levels were in fact responsible for inhibiting autoignition chemistry. These results should, however, be considered in light of the differences between counterflows on the one hand, and mixing layers and coflows on the other (Markides \& Mastorakos 2005). In a counterflow, the residence time is determined entirely by the inlet jet velocities and the geometry, limiting the time available for reaction as the reactants flow axially towards each other and then radially outwards. Furthermore, the level of $\chi$ experienced by the flow does not decay and remains at the level set by the velocities and initial separation of the jets. In a mixing layer, the total residence/reaction time available to the flow varies with the streamwise domain length, and $\chi$ decays as the reactants mix. This is more similar to coflows, where the total residence (mixing and reaction) time can always be made arbitrarily long by using a longer domain, and $\chi$ always decays.

Markides \& Mastorakos (2005) investigated experimentally the effects of the flow on the autoignition of pure and nitrogen-diluted $\mathrm{H}_{2}$ plumes and jets in a 'confined turbulent hot coflow' (CTHC) configuration where the fuel was released concentrically into the confined coflow of turbulent, electrically preheated air with bulk injection velocities between one and five times that of the coflow. Depending on the velocities, temperatures and composition of the two streams, localized autoignition kernels appeared at well-defined but random locations and instances and unsteady flamelets propagated from these kernels into the surrounding inhomogeneous mixture either: (i) extinguishing in the neighbourhood of the original autoignition site, or propagating 
over a short distance and giving rise to 'secondary' autoignition events ('random spots' regime); or propagating upstream to either (ii) create a lifted flame ('lifted flame' regime) or (iii) establish a flame anchored at the injector nozzle ('flashback' regime). These regimes were observed over a wide range of flow conditions, geometric parameters (turbulence generation grid sizes, injectors, etc.), and fuels including acetylene, ethylene and prevapourized $n$-heptane (Markides 2005; Markides, de Paola \& Mastorakos 2007; Markides \& Mastorakos 2008a).

In the random spots regime, the experiments showed that an increase in the bulk coflow air velocity $U_{a}$ results in an increase in the autoignition length from the injector $L_{i g n}$, and in the associated mean residence time until autoignition $\tau_{i g n}=L_{i g n} / V$, where $V$ is a suitably chosen mean advection velocity. For hydrogen, this conclusion was drawn for $\tau_{i g n} / \tau_{t} \approx 0.2-1.3$ (Markides 2005). The influence of $U_{a}$ on the flow and mixing fields in the CTHC geometry could only be investigated in cold conditions, whereby an effort was made to ensure dynamic and kinematic similarity with the autoigniting flows. Within the range of investigated conditions $\left(R e_{t}=u^{\prime} \ell_{I} / v=85-140\right)$, it was found that an increase in $U_{a}$ was accompanied by an increase in the turbulent axial velocity fluctuations $u^{\prime}$, and to a lesser extent by a reduction in the turbulent longitudinal integral length scale $\ell_{I}$. The mean mixture fraction field $\langle\xi\rangle$ remained unaffected (Markides 2005; Markides \& Mastorakos 2006). It was inferred that autoignition was inhibited by an increase in turbulence levels, and by implication by the higher scalar dissipation caused by the higher $u^{\prime}$ and smaller $\ell_{I}$.

In stagnant mixing layers, once successfully ignited from a spot a post-ignition flame always propagates to cover the whole mixing layer. The presence of strong advection, a situation that has not been simulated in the past, can alter this picture through the continuous replenishment of the reacting regions with cold or partially reacted fluid, which can in turn affect the dynamics and possible stabilization of lifted flames. A recent three-dimensional DNS study of ignited jet-type flows (hydrogen injected into a heated coflow), with detailed chemistry for the investigation, examined the interaction between autoignition and flame stabilization (Yoo, Sankaran \& Chen 2009). The autoignition and post-ignition flame propagation behaviour observed in the CTHC has some similarities with the characteristics of lifted flames generated by the introduction of fuel jets into vitiated coflows ('JVC'), where autoignition ahead of the strongly burning flame is important for flame stabilization (Dally, Karpetis \& Barlow 2002; Cabra et al. 2002, 2005; Wu, Masri \& Bilger 2006; Medwell, Kalt \& Dally 2007, 2008; Gordon, Masri \& Mastorakos 2008; Oldenhof, Tummers, van Veen \& Roekaerts 2010). The transient flame dynamics exhibited in both configurations are much more complex than those of conventional lifted non-premixed turbulent flames, including the mechanisms responsible for flame initiation by autoignition, as well as formation and stabilization (or lack thereof in the 'random spots' case of the CTHC).

Autoignition in a confined low-shear flow where the background turbulence rather than the fuel jet dominates the mixing process is relevant to combustors, such as the ducts of lean premixed prevapourized (LPP) gas turbines, and deserves further consideration. Despite the significant progress that has been made regarding the effects of turbulence on autoignition, a complete clarification is still lacking. It is necessary to collect detailed information that would enhance our understanding of the underlying physical processes. Experimentally, the harsh and extremely sensitive autoignition environment has prohibited the simultaneous probing of the underlying turbulent flow, mixing and reaction fields in hot conditions. According to Mastorakos (2009), only a handful of experiments exist with refined enough results to reveal something about turbulence-autoignition interactions. Guided by the CTHC setup 
and the experimentally determined range of conditions for the random spot regime, numerical experiments were performed in order to: $(a)$ obtain the detailed description of the flow, mixing and reactive processes leading to random spots; $(b)$ analyse the simulation data to identify the characteristics of autoignition in the presence of strong advection; and $(c)$ provide data which can be used for model development and validation.

The paper is organized as follows: $\S 2$ presents the problem setup, including the geometry, the flow conditions and the employed numerical methodology employed, while $\S 3$ discusses autoignition in homogeneous reactors and the choice of the chemical mechanism. Following the characterization of the velocity and mixing field in $\S 4$, the main results and findings with respect to qualitative observations of autoignition-related phenomena, and observations of autoignition in physical and mixture fraction space are presented in $\S 5$. Concluding remarks are provided, summarizing the main findings, in $\S 6$.

\section{Problem definition and numerical methodology}

The simulations were performed with a low-Mach-number reactive flow solver (Kerkemeier 2010) which is based on the highly scalable, incompressible spectral element flow solver nek5000 (Fischer, Lottes \& Kerkemeier 2011). The conservation equations for total mass, species, momentum and energy complemented by the equation of state (ideal gas law) in the low-Mach-number limit can be found in (Kerkemeier 2010; Pizza et al. 2010). Time advancement is performed using a highorder splitting scheme (Tomboulides, Lee \& Orzag 1997) which decouples the highly nonlinear and stiff thermochemistry subsystem (energy and species equations) from the hydrodynamic subsystem (momentum equation) through a third-order extrapolation scheme (EXT). The backward differentiation formula (BDF)-based stiff ordinary differential equation solver CVODE (Hindmarsh et al. 2005) is used to integrate the thermochemistry system. The third-order temporal integration of the hydrodynamic subsystem is based on a semi-implicit formulation (EXT/BDF) treating the nonlinear advection term explicitly (Deville, Fischer \& Mund 2002; Kerkemeier 2010). The thermodynamics properties, as well as the detailed chemistry and transport based on the mixture-average transport properties are provided by optimized routines compatible with CHEMKIN (consult Kerkemeier (2010) for further details).

\subsection{Problem definition}

Referring to figure 1 , the computational domain is a cylinder of diameter $D=16 \mathrm{~mm}$ and total height $H=55 \mathrm{~mm}$. The mesh is constructed by extruding and joining twodimensional slices of two conforming grids, one for the section upstream of the injector exit (figure $1 b$ ) and one for the main flow section (figure 1c). The domain is discretized into 963264 spectral elements, within each of which the solution is approximated by a three-dimensional tensor-product based on fourth-order Lagrange polynomials defined on Gauss-Lobatto quadrature points resulting in a total of more than 120 million discretization points. In the axial direction, the grid is refined locally to resolve the fuel injector boundary layer and the autoignition region in the downstream half of the domain.

The fuel, hydrogen with a mass fraction of $Y_{H_{2}}=0.14$ diluted in nitrogen, enters the domain through a circular injector nozzle of inner diameter $d_{i}=2 r_{i}=2.25 \mathrm{~mm}$ at a height $z=-4.5 \mathrm{~mm}$, where a parabolic fuel inflow velocity profile is imposed. The laminar fuel stream issues with bulk velocity $U_{f}=26 \mathrm{~m} \mathrm{~s}^{-1}$ and 
(a)

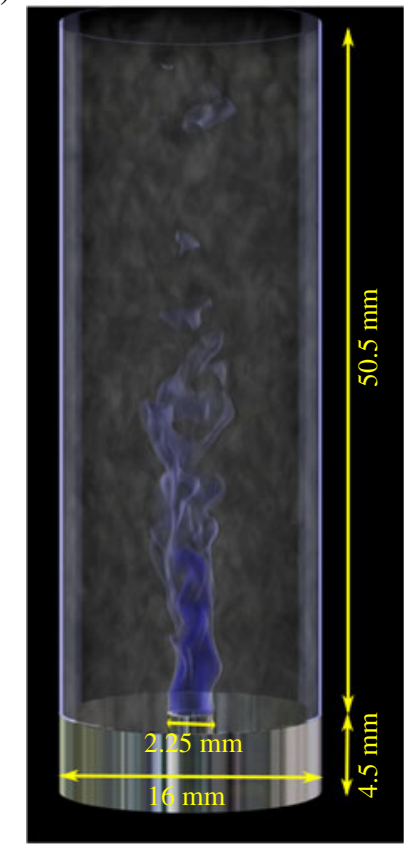

(b)

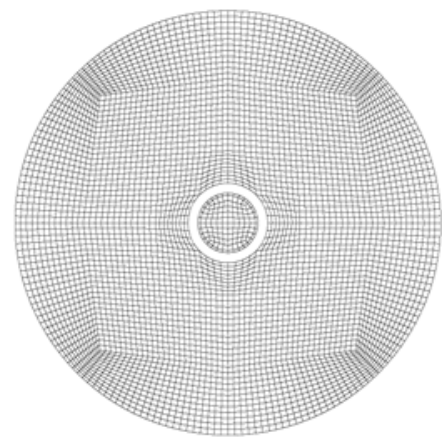

(c)

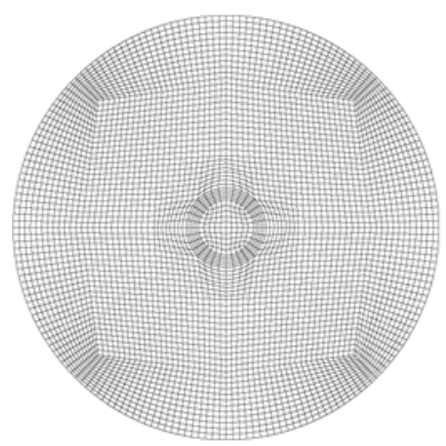

FIgURE 1. (Colour online) (a) Schematic of the numerical domain. Horizontal slices of the spectral element skeleton $(b)$ upstream of the injector and $(c)$ in the main flow section.

temperature $T_{f}=855 \mathrm{~K}$ into the coflowing hot turbulent air stream. In order to avoid prescribing the mixture fraction profile and therefore an a priori scalar dissipation rate profile at the fuel nozzle exit, the air is separated from the fuel by a concentric injector tube with height $h=4.5 \mathrm{~mm}$ and outer diameter $d_{o}=3.0 \mathrm{~mm}$. The air enters the domain with a bulk velocity $U_{a}=U_{f}=U=26 \mathrm{~m} \mathrm{~s}^{-1}$ and temperature $T_{a}=945 \mathrm{~K}$ through the inflow boundary at $z=-4.5 \mathrm{~mm}$. A top-hat mean air velocity profile is imposed at the inflow boundary, which via a hyperbolic tangent profile drops smoothly to zero at the injector wall.

Unlike in the experiments where the turbulence was manipulated by changing the background mean flow velocity (i.e. the velocity fluctuations $u^{\prime}$ were increased by increasing $U_{a}$ ), two levels of turbulence intensity, are considered here but with the same background flow velocity. A synthetic turbulence generation method (Klein, Sadiki \& Janicka 2003) is used to compute correlated velocity fluctuations with integral length scale $\ell_{I}=3 \mathrm{~mm}$, presuming a fully developed homogeneous isotropic energy spectrum. Using Taylor's hypothesis, velocity fluctuations with turbulence intensity $I=u^{\prime} / U$ are superimposed on the mean inlet air velocity at the coflow inflow boundary. Two turbulence intensities are considered (table 2): (i) a low $I_{i}=0.15$ case representative of the experimental conditions in the CHTC (Markides \& Mastorakos 2011); and (ii) a high $I_{i}=0.25$ case. Based on these values a turbulent integral time scale (or eddy turnover time) $\tau_{t}=\ell_{I} / u^{\prime}$ can be defined, which over the injector length increases from 0.8 to $1.0 \mathrm{~ms}$ in the low $I_{i}$, and from 0.5 to $0.6 \mathrm{~ms}$ in the high $I_{i}$ case.

At the air inflow boundary, the temperature is uniform and steady, while a zero-flux condition is imposed on the species mass fractions. Zero-slip, adiabatic and zero-flux 
Parameter

$D$

$H$

$h$

$d_{i}=2 r_{i}$

$d_{o}$

$U_{f}=U$

$T_{f}$

$Y_{H_{2}}$

$U_{a}=U$

$T_{a}$

$t_{r}=r_{i} / U$

$t_{f}=H / U$

$t_{i}=h / U$

$t_{T}=4.67 t_{f}$
Description

Domain diameter

Total domain height (including injector length)

Extent of domain upstream of injector nozzle

Inner fuel nozzle diameter

Outer fuel nozzle diameter

Bulk injected fuel velocity

Fuel temperature

Hydrogen mass fraction in injected fuel stream

Bulk coflow air velocity

Air temperature

Convective reference time

Total flow-through time

Injector flow-through time

Simulated time after fuel injection
Value

$16 \mathrm{~mm}$

$55 \mathrm{~mm}$

$4.5 \mathrm{~mm}$

$2.25 \mathrm{~mm}$

$3.0 \mathrm{~mm}$

$26 \mathrm{~m} \mathrm{~s}^{-1}$

$855 \mathrm{~K}$

0.14

$26 \mathrm{~m} \mathrm{~s}^{-1}$

$945 \mathrm{~K}$

$43.3 \mu \mathrm{s}$

$2.12 \mathrm{~ms}$

$0.17 \mathrm{~ms}$

9.9 and $11.5 \mathrm{~ms}$

TABLE 1. Geometry, flow conditions and simulation parameters.

Parameter

$I_{i}=u^{\prime} / U(z=-h)$

$I_{0}=u^{\prime} / U(z=0)$

$\ell_{I}$

$$
\begin{aligned}
& R e_{t}=u^{\prime} \ell_{I} / v \\
& \tau_{t, i}=\ell_{I} / u^{\prime}(z=-h) \\
& \tau_{t, 0}=\ell_{I} / u^{\prime}(z=0) \\
& \eta_{K}=\left(v^{3} / \epsilon\right)^{1 / 4} \\
& \tau_{K}=(v / \epsilon)^{1 / 2} \\
& \mathrm{~d} x \\
& \mathrm{~d} t
\end{aligned}
$$

\section{Description}

Coflow air turbulence intensity at inlet Coflow air turbulence intensity at injection plane

Coflow air integral length scale at domain inlet

Turbulent Reynolds number at injection

Turbulent eddy turnover time at inlet

Turbulent eddy turnover time at injection

Kolmogorov length scale at injection

Kolmogorov time scale at injection Mesh spacing Time step
Case value

Low $I$ High $I$

$\begin{array}{ll}0.15 & 0.25 \\ 0.13 & 0.21\end{array}$

$3 \mathrm{~mm}$

100

170

$0.77 \mathrm{~ms} \quad 0.46 \mathrm{~ms}$

$0.96 \mathrm{~ms} \quad 0.58 \mathrm{~ms}$

$0.11 \mathrm{~mm} \quad 0.07 \mathrm{~mm}$

$0.11 \mathrm{~ms} \quad 0.05 \mathrm{~ms}$

$<0.06 \mathrm{~mm}$

$0.43 \mu \mathrm{s}$

TABLE 2. Turbulence parameters used in the low- and high-turbulence-intensity simulations; also including the spatial and temporal resolution of the simulations.

boundary conditions are used for the velocity, temperature and species, respectively, on the inside and outside injector walls. Finally, a moving-wall boundary condition for the velocity, and zero-flux conditions for the temperature and species are used on the lateral boundary at $r=8 \mathrm{~mm}$, while a zero-gradient condition is imposed on all variables at the outflow boundary. Table 1 summarizes important problem variables and parameters.

The length of the injector also allows the synthetic turbulence to develop before the air and fuel streams come into contact. By the time the flow has reached the injector exit, the turbulence intensity levels have decayed to $I_{0}=0.13$ and 0.21 , in the low and high $I_{i}$ case, respectively. At the air inflow plane, the corresponding turbulent Reynolds numbers for the low and high turbulence intensities are $R e_{t}=u^{\prime} \ell_{I} / v=105.4$ and 175.7 (based on the dynamic viscosity of air $v_{\text {air }}=1.11 \mathrm{~cm}^{2} \mathrm{~s}^{-1}$ at $945 \mathrm{~K}$ ). The Kolmogorov length $\eta_{K}=\left(\nu^{3} / \epsilon\right)^{1 / 4}$ and time scales $\tau_{K}=(\nu / \epsilon)^{1 / 2}$ based on the energy dissipation 
rate extracted from the simulation data are 0.11 and $0.07 \mathrm{~mm}$ and 0.11 and $0.05 \mathrm{~ms}$, respectively.

The non-uniform mesh spacing determined by the number of spectral elements and the polynomial order used satisfies strictly $\max (\mathrm{d} x)=0.06 \mathrm{~mm}$. It is thus sufficiently fine to resolve all turbulence scales (Moin \& Mahesh 1998), with $\mathrm{d} x<0.9 \eta_{K}$ in the high $I_{i}$ case and $\mathrm{d} x<0.6 \eta_{K}$ in the low $I_{i}$ case. It is also fine enough to resolve all mixing scales (i.e. the Batchelor scale $\eta_{B}=\left(\nu D^{2} / \epsilon\right)^{1 / 4}=\left(\eta_{K} / S c^{1 / 2}\right)^{1 / 4}$ and the Obukhov-Corrsin scale $\left.\eta_{O C}=\left(D^{3} / \epsilon\right)^{1 / 4}=\left(\eta_{K} / S c^{3}\right)^{1 / 4}\right)$, which are larger than $\eta_{K}$ since the Schmidt number is $S c=v / D<1$. The integration time step for the flow was $\mathrm{d} t=0.01 t_{r}$, where the convective reference time scale is $t_{r}=r_{i} / U=43.3 \mu \mathrm{s}$, sufficiently smaller than the shortest flow time scale $\tau_{K}$. During the fixed $\mathrm{d} t$ for the hydrodynamic part, the time step for the thermochemistry integration is automatically adapted by CVODE in order to keep the local error below a value determined by the user-specified absolute and a relative tolerances of $10^{-10}$ and $10^{-6}$, respectively. The spatial and temporal resolution independence of the solution was verified by repeating the calculation for the initial $2.6 t_{f}$ using sixth-order polynomials and time step $\mathrm{d} t / 2$. Only minor differences were uncovered with respect to the integral of the heat release over the entire domain, which was found to be a sensitive indicator of the temporal evolution of the flow, mixing and chemistry, as well as the instantaneous profiles of all variables and the structure of the autoignition spots (AISs).

The simulations were performed as follows: initially, pure $\mathrm{N}_{2}$ was injected in a domain filled with air and the equations were integrated for $t_{0}=2 t_{f}$ to allow the flow to develop; $t_{f}=H / U=2.12 \mathrm{~ms}$ is the flow-through time. At $t=0$, hydrogen was introduced in the fuel stream so that $Y_{H_{2}}=0.14$. Following injection, the fuel mixed with the hot coflowing air stream and started reacting as it filled the domain. The total time simulated following fuel injection was $t_{T}=4.7 t_{f}$ and $5.4 t_{f}$ for the low and high $I_{i}$ cases, respectively. All physical and simulation parameters are summarized in tables 1 and 2. Unless otherwise noted, in the rest of the paper all quantities will be presented in non-dimensional units with respect to the fuel nozzle radius $r_{i}$, the bulk velocity at the inlet $U=U_{f}=U_{a}$, and the mixture properties at the fuel nozzle.

As discussed in the next section, four chemical mechanisms were tested, and the detailed mechanism of Li et al. (2004) containing 9 chemical species $\left(\mathrm{H}_{2}, \mathrm{O}_{2}, \mathrm{H}_{2} \mathrm{O}, \mathrm{H}\right.$, $\mathrm{O}, \mathrm{OH}, \mathrm{HO}_{2}, \mathrm{H}_{2} \mathrm{O}_{2}$ and $\mathrm{N}_{2}$ ) and 21 reversible elementary reactions was employed in the simulations.

\section{Homogeneous autoignition and pre-ignition chemistry}

Consider first homogeneous adiabatic constant pressure reactors with an initial mixture composition defined based on inert mixing of fuel with air, whose composition is characterized completely by the mixture fraction $\xi$

$$
Y_{k, 0}=\xi Y_{k, f}+(1-\xi) Y_{k, a}
$$

where the subscripts ' $f$ ' and ' $a$ ' refer to values in the fuel and air streams, respectively. The initial temperature in each reactor $T_{0}$ can then be computed from the initial enthalpy $H_{0}$ of the corresponding mixture,

$$
H_{0}\left(T_{0}\right)=\xi H_{f}\left(T_{f}\right)+(1-\xi) H_{a}\left(T_{a}\right)
$$

The 'homogeneous autoignition delay time' $\tau_{\text {ign, }, 0}$, defined by the time of the maximum heat release rate, depends solely on the chemical kinetics and the initial conditions. Using the detailed mechanism of $\mathrm{Li}$ et al. (2004), $\tau_{i g n, 0}$ differs only slightly 

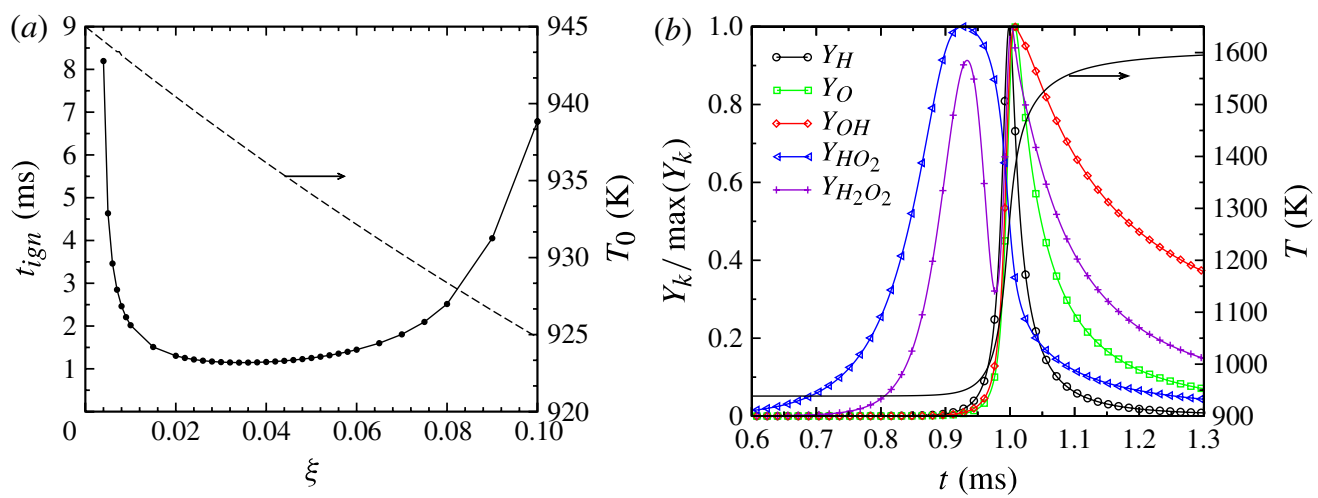

FIgURE 2. (Colour online) Homogeneous autoignition with the detailed mechanism of Li et al. (2004). (a) Autoignition delay time $\tau_{i g n, 0}$ as a function of mixture fraction in a homogeneous reactor (solid line with filled circles); the corresponding initial mixture temperature $T_{0}$ is shown by the dashed line. $(b)$ Temporal evolution of temperature and normalized species mass fraction during autoignition of the mixture corresponding to $\xi_{M R, 0}=0.035$.

from the global minimum for $0.02<\xi<0.06$ (figure $2 a$ ), while outside this range it increases steeply in both directions. A representative value of the "homogeneous most reactive mixture fraction' $\xi_{M R, 0}=0.035$ is associated with $\tau_{i g n, 0}=1.1 \mathrm{~ms}$. Owing to the dominant effect of temperature on the chemical reaction rates and the higher initial temperature of the air side $(\xi=0), \xi_{M R, 0}$ is significantly leaner than the stoichiometric value $\xi_{s t}=0.17$

Indicating the chemical processes associated with autoignition, figure $2(b)$ shows the normalized mass fractions of various species for the mixture corresponding to $\xi_{M R, 0}$. The hydroperoxyl radical $\mathrm{HO}_{2}$ is produced before the hydroxyl radical $\mathrm{OH}$, and the rise of $\mathrm{OH}$ appears almost concurrently with the rapid temperature increase associated with thermal runaway. A delay of approximately $0.08 \mathrm{~ms}$ exists between the peaks of $Y_{\mathrm{HO}_{2}}$ and $Y_{\mathrm{OH}}$. Hence, $\mathrm{HO}_{2}$ can be used as an indicator of the pre-ignition kinetics, and $\mathrm{OH}$ as an indicator of the thermal runaway and a marker of the flame location. The observation concerning $\mathrm{HO}_{2}$ is consistent with previous studies, which have shown that this radical is important in initiating autoignition upstream of the flame base (Yoo et al. 2009) and hence a good precursor for the $\mathrm{H}_{2}$ pre-ignition chemistry, irrespective of the value of $\xi_{M R}$ (Echekki \& Chen 2003).

The sensitivity of the autoignition delay times on the chemical mechanism shown in figure 3 was examined using four detailed $\mathrm{H}_{2} / \mathrm{O}_{2}$ schemes (Yetter, Dryer \& Rabitz 1991; del Álamo, Williams \& Sánchez 2004; Conaire et al. 2004; Li et al. 2004). Although a minimum $\tau_{i g n, 0}$ is observed for all mechanisms, significant differences are found, especially between the more recent mechanisms and the older one. At low temperatures where there is a larger uncertainty in the reaction rate constants, pronounced deviations exist even for the recent kinetic schemes.

\section{Velocity and mixing field characterization}

In this section we focus on the analysis of the velocity and mixing field data obtained in the low-turbulence-intensity $\left(I_{i}=0.15\right.$ and $\left.I_{0}=0.13\right)$ case, which is closer to the conditions of the corresponding experiments in the CTHC where $I_{0} \approx 0.12$ at 


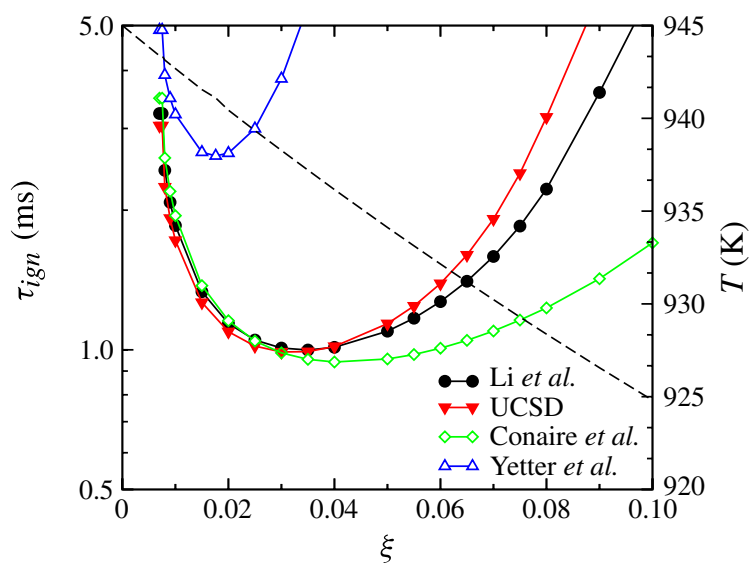

FIGURE 3. (Colour online) Autoignition delay time in a homogenous reactor $\tau_{i g n, 0}$ as a function of mixture fraction for the selected mechanism of Li et al. (2004) (filled circles), and the mechanisms of Yetter et al. (1991) (open upper triangles), del Álamo et al. (2004) (filled lower triangles), Conaire et al. (2004) (open diamonds). The initial mixture temperature is shown by the dashed line.

the fuel injection plane away from the walls (Markides 2005; Markides \& Mastorakos 2005).

\subsection{Velocity field}

Since the bulk velocities of the fuel $U_{f}$ and air streams $U_{a}$ are equal, strong mean velocity gradients are absent and far enough from the nozzle the initially laminar and axisymmetric fuel plume evolves in an environment that is approximated well by a spatially decaying homogenous isotropic turbulent flow field. Figure 4(a) shows the mean axial velocity $\left\langle u_{z}\right\rangle$ distribution on a $x-z$ plane passing through the jet axis. The dimensionless radial profiles of $\left\langle u_{z}\right\rangle$ plotted in figure $4(b)$ show that the initially parabolic velocity profile associated with the laminar, low-momentum fuel stream released at the exit of the injector nozzle (solid line at $z=0.1$ ) decays downstream, as it imparts its momentum to the wake region formed close to the injector walls and the recirculation zone downstream of the finite-thickness injector tube wall. This process is driven by the velocity gradients and the background turbulence in the coflowing air stream, and by $z \approx 15$ the mean flow field becomes nearly uniform.

The axial decay of the mean $\left\langle u_{z}\right\rangle$ (solid line) and variance $\left\langle u_{z}^{\prime 2}\right\rangle$ (dashed line) of the axial velocity component along the centreline are shown in figure $4(c)$. For $z \gtrsim 25$ the combustion process causes an acceleration in the mean flow, as expected due to gas expansion, and reduces the magnitude of the velocity fluctuations. Specifically, $\left\langle u_{z}\right\rangle$ increases by $20 \%$, while $\left\langle u_{z}^{\prime 2}\right\rangle$ decreases from $\sim 0.07$ to 0.05 in the region $25 \lesssim z \lesssim 30$.

Favre averaging, defined as $\{\phi\}=\langle\rho \phi\rangle /\langle\rho\rangle$ for a field $\phi$, can be employed in order to eliminate the effect of density variations. Figure 5 shows the normalized centreline profiles of the Favre-averaged turbulent kinetic energy $\{k\} /\{U\}^{2}$ where $k=\sum_{i} u_{i}^{\prime 2} / 2$, and the normal stresses $\left\{u_{i}^{\prime 2}\right\} /\{U\}^{2}, i=x, y, z$. Moving downstream from the injector where the flow is laminar and $\{k\}=0,\{k\}$ increases initially reaching a peak value at $z \approx 6.7$ and then decays downstream. Consistent with figure 4(c), the heat released from combustion has a dampening effect on the turbulent kinetic energy over the region from $z \approx 27$ to 31 . The three normal stresses become nearly isotropic for $z>8$ 
(a)

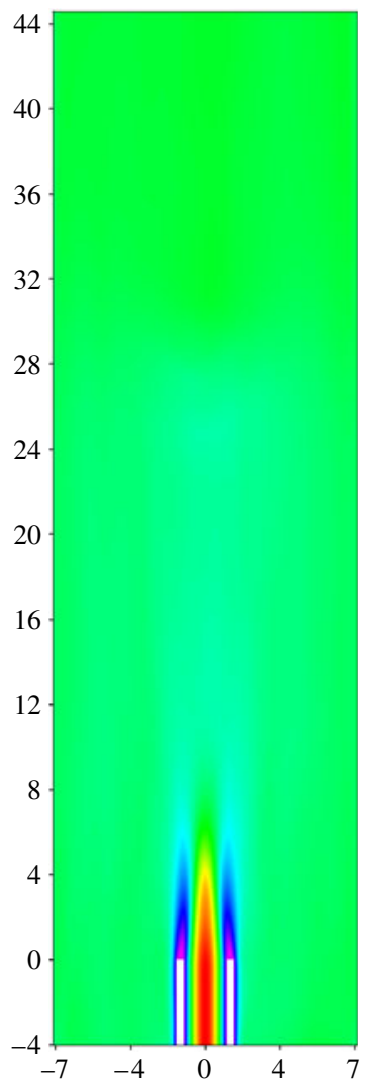

(b)

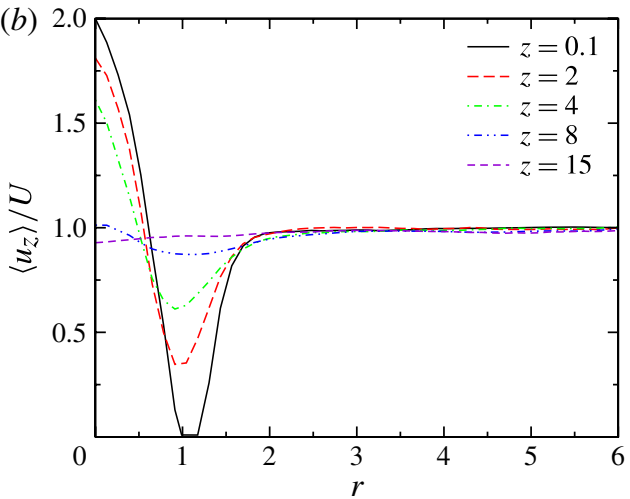

(c)

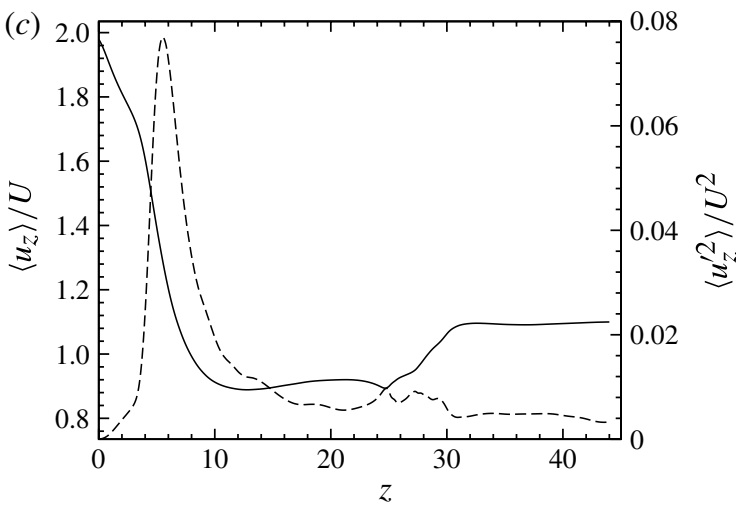

FIgURE 4. (Colour online) Mean axial velocity field. (a) Distribution of the axial mean velocity $\left\langle u_{z}\right\rangle(x, z)$ in a $x-z$ plane passing through the centreline of the flow. $(b)$ Radial profiles of $\left\langle u_{z}\right\rangle$ at different axial locations. (c) Centreline profiles of the mean $\left\langle u_{z}\right\rangle$ (solid line) and variance $\left\langle u_{z}^{\prime 2}\right\rangle$ (dashed line) of the axial velocity component.

(figure 5b). Their downstream decay follows a power law expression $\left\{u_{i}^{\prime 2}\right\} /\{U\}^{2} \propto z^{-n}$ with $n=1.57$ downstream of the immediate vicinity of the nozzle $(z>8)$, and $n=1.41$ over the entire region shown in the figure. These values of $n$ are well within literature values for homogeneous isotropic grid-generated turbulence (Mohamed \& LaRue 1990; Huang \& Leonard 1994; Pope 2000). The probability density functions (p.d.f.s) of the square of the velocity fluctuations, $P\left(u_{i}^{\prime 2}\right)$ reveal that the velocity fluctuations in all three directions are well described by a Gaussian distribution, as expected for turbulent free shear flow (figure 6).

\subsection{Mixing field}

\subsubsection{Mixture fraction}

Figure 7 shows volume-rendered images of $(a)$ an instantaneous and $(b)$ the timeaveraged isosurfaces of $\xi_{s t}=0.17$ (orange) and $\xi=0.05$ (cyan) as well as streamwise slices $\left(x-z\right.$ planes) of $(c)$ the mean $\langle\xi\rangle$ and $(d)$ variance $\left\langle\xi^{\prime 2}\right\rangle$ of the mixture fraction. Superimposed in figure $7(c, d)$ are the projected on the $x-z$ plane locations of the AISs (crosses) which will be discussed in the next section. The mean stoichiometric mixture fraction isosurface closes at a height that is significantly lower than that of the 

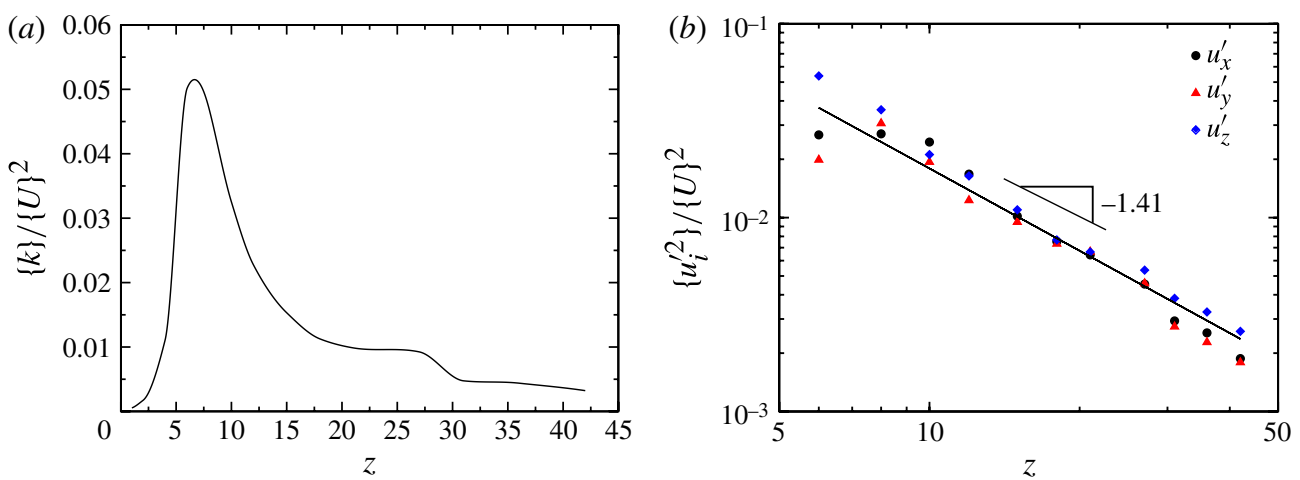

FIGURE 5. (Colour online) Profiles of normalized Favre-averaged $(a)$ centreline decay of turbulent kinetic energy $\{k\}$ and $(b)$ centreline decay of normal Reynolds stresses: $\left\{u_{x}^{\prime 2}\right\}$ (circles), $\left\{u_{y}^{\prime 2}\right\}$ (triangles), $\left\{u_{z}^{\prime 2}\right\}$ (diamonds), also showing a power law fit proportional to $z^{-1.41}$ (solid line).

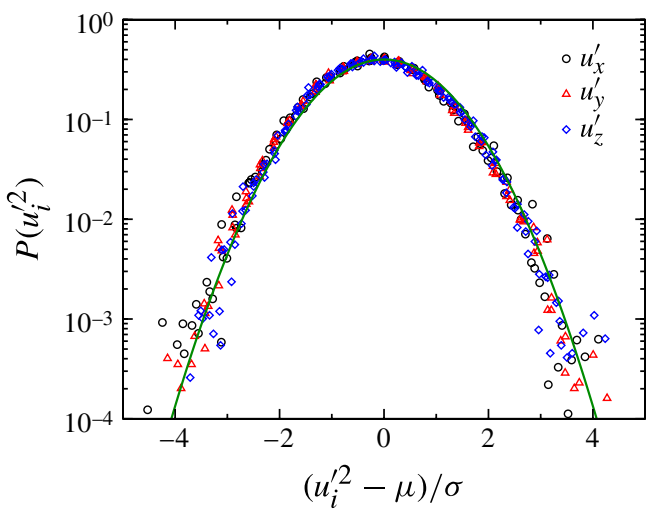

FIGURE 6. (Colour online) Normalized p.d.f. of velocity fluctuations $u_{x}^{\prime 2}$ (circles), $u_{y}^{\prime 2}$ (triangles), $u_{z}^{\prime 2}$ (diamonds), compared with a Gaussian profile (solid line). Here, $\mu$ and $\sigma$ are the mean and standard deviation of $u_{i}^{\prime 2}$, respectively.

spots, which therefore appear at compositions significantly leaner than stoichiometric. On the other hand, the most reactive mixture fraction is so lean that its mean isosurface extends over the whole domain height, and much farther downstream from the autoignition region.

Along the centreline, the mean mixture fraction $\langle\xi\rangle_{r=0, z}$ decays at a rate of $z^{-1.5}$ sufficiently far away from the nozzle $\left(z>3\right.$ or $\left.z / \ell_{I}>1\right)$ and drops below the stoichiometric value at $z \approx 15$ (figure $8 a$ ). The decay rate falls between the value for a self-preserving axisymmetric jet that decreases as $z^{-1}$ (Pope 2000) and Gaussian dispersion from a point source at short lengths for which the decay rate is proportional to $z^{-2}$ (Sutton 1932). In the former, the diffusion process is driven by the shear (velocity gradients) set up between the high-momentum jet and the surrounding atmosphere, whereas in the latter it is driven by the turbulence in the background flow upstream of the low-momentum release. Note here that according to Taylor's diffusion 
(a)

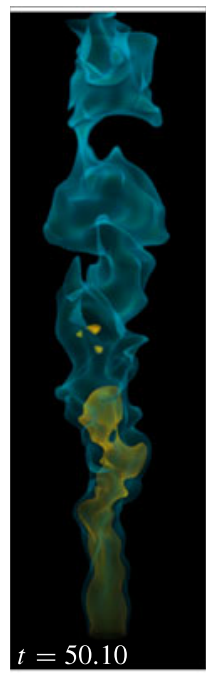

(b)

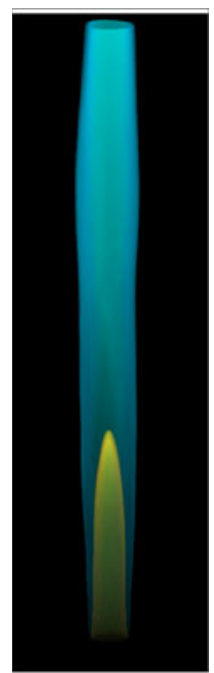

(c)

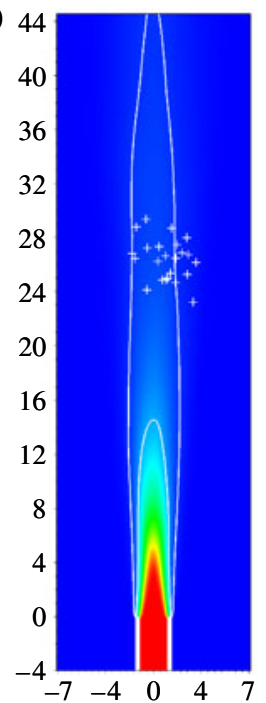

(d)

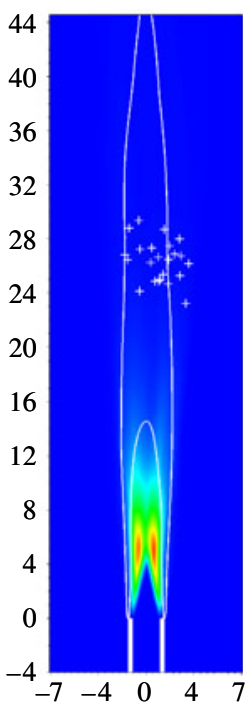

FIGURE 7. (Colour online) Volume renderings of $(a)$ instantaneous of stoichiometric mixture fraction $\xi_{s t}=0.17$ (orange in the lower part of the domain) and $\xi=0.05$ (cyan extending over the whole domain) isosurfaces, $(b)\langle\xi\rangle=\xi_{s t}=0.17$ (orange) and $\langle\xi\rangle=0.05$ (cyan) isosurfaces. Centreline $x-z$ slice of $(c)$ mean mixture fraction $\langle\xi\rangle$ and $(d)$ mixture fraction variance $\left\langle\xi^{\prime 2}\right\rangle$. In $(c, d)$ the inner and outer white lines are the stoichiometric $\xi_{s t}=0.17$ and the $\langle\xi\rangle=0.05$ isocontour, respectively. The white crosses mark the AIS locations projected on the $x-z$ plane.

(a)

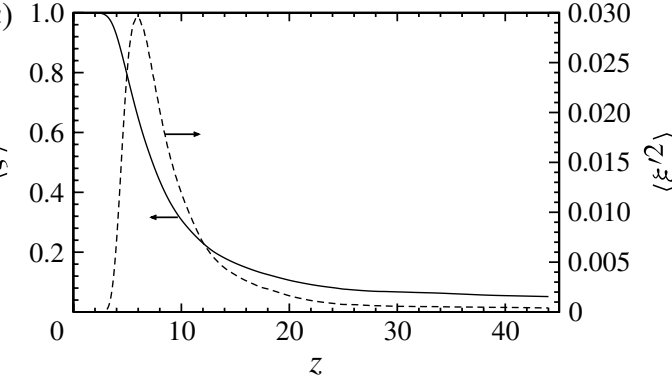

(b)

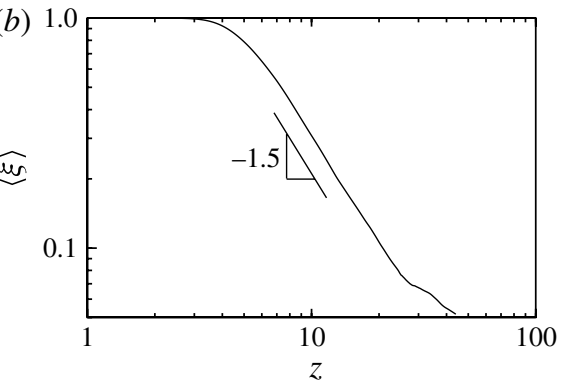

FIGURE 8. (a) Centreline profile of the mean $\langle\xi\rangle_{r=0, z}$ and variance $\left\langle\xi^{\prime 2}\right\rangle_{r=0, z}$ of the mixture fraction $\xi$. (b) $\log -\log$ profile of $\langle\xi\rangle_{r=0, z}$.

theory for Gaussian axisymmetric plumes, one would expect the mean concentration profile along the centreline (and, hence, the axial profile of $\langle\xi\rangle_{r=0, z}$ ) to obey a $z^{-2}$ power law at short distances from the fuel point source and a $z^{-1}$ power law at longer distances. The demarkation between short and long distances from the nozzle is based on the order of magnitude comparison between the characteristic plume width $\sigma_{r}=r_{1 / 2} / \sqrt{2 \ln (2)}$ and the outer turbulence length scales. Here, $r_{1 / 2}$ is the plume width at which $\langle\xi\rangle$ decreases to half of its centreline value and the relevant turbulent length scale is $\ell_{I}$. In the current case we would expect short-length behaviour, for which $\sigma_{r} \approx I_{i} z$ at $\sigma_{r} \ll \ell_{I}$, and long-length behaviour for which $\sigma_{r}^{2} \approx 0.2 I_{i} \ell_{I} z$ at $\sigma_{r} \gg \ell_{I}$. The 

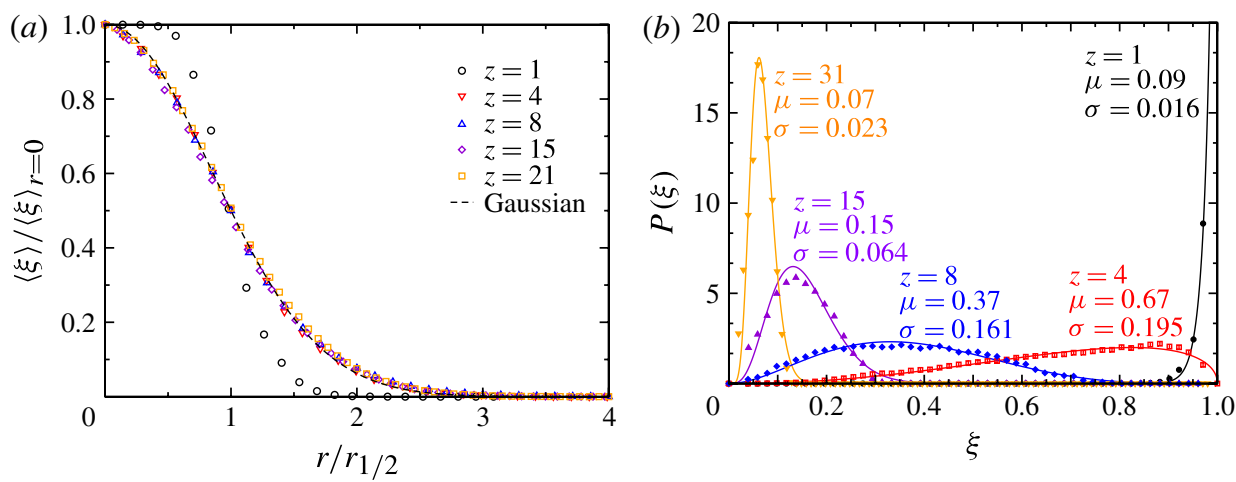

FIGURE 9. (Colour online) (a) Normalized radial mean mixture fraction versus radius normalized by the plume half-width radius $r_{1 / 2} ;(b)$ p.d.f. of mixture fraction $P(\xi)$ at various axial distances $z$ along $r=0.5$ extracted from the DNS results (open circles). The solid lines are a posteriori determined beta distributions $P_{\beta}(\xi)$ (equation (4.2)) using the local mean $\mu$ and standard deviation $\sigma$ values indicated on the plot.

two conditions correspond to $z \ll 30$ and $z \gg 100$, respectively, so that short-length behaviour is expected. Returning to figure $8(b)$, at long distances $(z \gtrsim 25)$ the decay rate decreases as a result of autoignition.

The radial profile of the mean mixture fraction $\langle\xi\rangle_{x, z}(r)$ normalized by the centreline value $\langle\xi\rangle_{r=0, z}$ is shown in figure $9(a)$ at different streamwise locations. In agreement with mixing field measurements in the CTHC under similar conditions (Markides 2005; Markides \& Mastorakos 2008b, 2011), the mean mixture fraction profiles have a self-similar shape for $z / \ell_{I}>1$ that can be described well by a Gaussian function,

$$
\frac{\langle\xi\rangle_{r, z}}{\langle\xi\rangle_{r=0, z}}=\exp \left\{-\frac{1}{2}\left(\frac{r}{\sigma_{r}}\right)^{2}\right\}=\exp \left\{-\ln 2\left(\frac{r}{r_{1 / 2}}\right)^{2}\right\} \text {. }
$$

Similar observations were reported e.g. by Lemoine et al. (2000) and Nakamura, Sakai \& Miyata (1987) for a plume in grid-generated turbulence.

The p.d.f. of the mixture fraction $P(\xi)$ in figure $9(b)$ is another way to describe the degree of mixing. Moving along $r=0.5$ axially downstream from the fuel source (slightly off-axis, but initially still inside the injector), the p.d.f. changes from a delta function at $\xi=1$ (fuel) gradually towards a delta function corresponding to the well-mixed $(\mathrm{WM})$ mixture with $\xi_{W M} \approx 0.008$. The p.d.f.s are found to be in excellent agreement with the a posteriori evaluated two-parameter beta p.d.f.

$$
P_{\beta}(\xi)=\xi^{\alpha-1}(1-\xi)^{\beta-1} \frac{\Gamma(\alpha+\beta)}{\Gamma(\alpha) \Gamma(\beta)},
$$

where $\Gamma(x)$ is the gamma function, and the two parameters $\alpha=\mu \gamma$ and $\beta=(1-\mu) \gamma$ are evaluated from the local mixture fraction mean and variance $\mu$ and $\sigma^{2}$ extracted from the data and $\gamma=\mu(1-\mu) / \sigma^{2}-1$.

\subsubsection{Scalar dissipation rate}

The centreline profile of the dimensionless mean scalar dissipation rate (figure 10a) $\langle\chi\rangle$ peaks at $z \approx 5.5$, and subsequently decays very quickly as $z^{-4.4}$ (figure $10 b$ ), so that autoignition appears in a region $(22<z<29$, see table 4) of very low scalar 

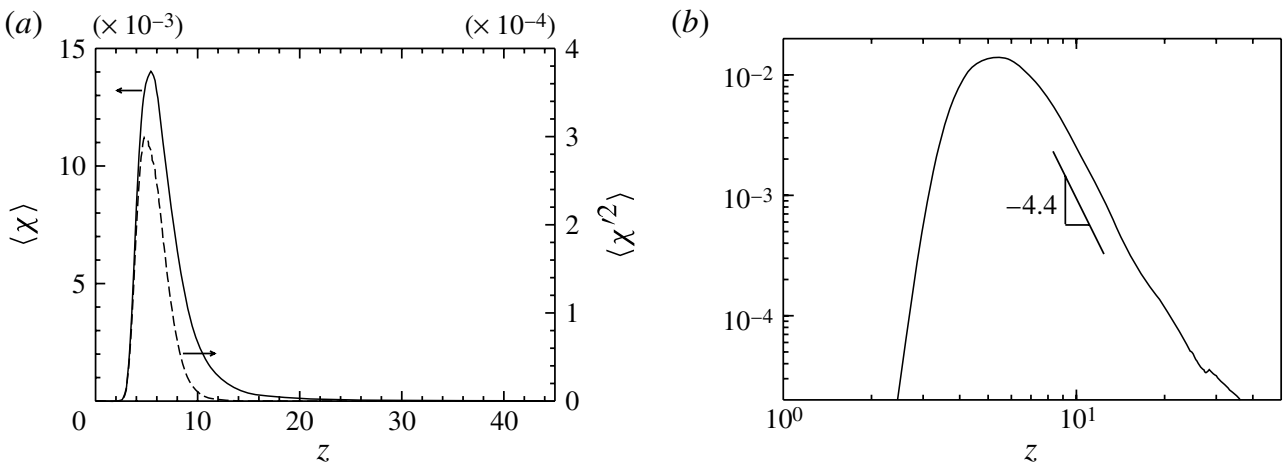

FIgURE 10. (a) Centreline profile of the mean $\langle\chi\rangle_{r=0, z}$ and variance $\left\langle\chi^{\prime 2}\right\rangle_{r=0, z}$ of the unconditional scalar dissipation rate $\chi .(b) \log -\log$ profile of $\langle\chi\rangle_{r=0, z}$ in $(a)$, also indicating a power law relation proportional to $z^{-4.4}$.

dissipation. Any effect of $\chi$ on autoignition would be through the history of conditions experienced by the reacting mixture from the injector to the AISs locations.

The average conditional scalar dissipation rate $\langle\chi \mid \xi\rangle$ is of greater interest than its unconditioned counterpart because of its importance in the interpretation of the autoignition process and its use in various modelling approaches. Figure 11 shows scatter plots of the conditional scalar dissipation rate $\chi \mid \xi$ in mixture fraction space, and the corresponding conditional mean $\langle\chi \mid \xi\rangle$ (solid lines) compiled over time at different points in physical space. The solid curves of $\langle\chi \mid \xi\rangle$ in figure 11 are found to be similar to the bell-shaped functions of $\chi(\xi)$ that result from the amplitude mapping closure (AMC) model given by

$$
\frac{\langle\chi \mid \xi\rangle}{\langle\chi\rangle}=\frac{G(\xi)}{\int_{0}^{1} G(\eta) P_{\beta}(\eta) \mathrm{d} \eta},
$$

for an a priori known $\langle\chi\rangle$, where

$$
G(\xi)=\exp \left\{-2\left[\operatorname{erf}^{-1}(2 \xi-1)\right]^{2}\right\},
$$

erf ${ }^{-1}$ being the inverse error function. Proposed by O'Brien \& Jiang (1991), the AMC model represents steady mixing in a laminar counterflow configuration and requires that the extrema of the mixture fraction (i.e. some unmixed fluid) are always present at zero (air) and unity (fuel). The AMC formulation provides an accurate prediction of $\langle\chi \mid \xi\rangle$ in turbulent inhomogeneous reacting flow conditions (Kim 2004) and was successfully employed in Reynolds averaged Navier-Stokes (RANS) simulations of the CTHC experiments by Markides et al. (2007). The AMC model (dashed lines) captures the conditional mean $\langle\chi \mid \xi\rangle$ (solid lines) reasonably well (figure 11). The deviations become larger farther downstream of the nozzle, but the scalar dissipation rate value there has decreased by an order of magnitude.

The scatter plots show that the conditional scalar dissipation rate is broadly distributed about the conditional mean. The corresponding p.d.f. $P(\chi \mid \xi)=$ $P(\chi, \xi) / P(\xi)$ describing the magnitude of the fluctuations about the conditional mean 

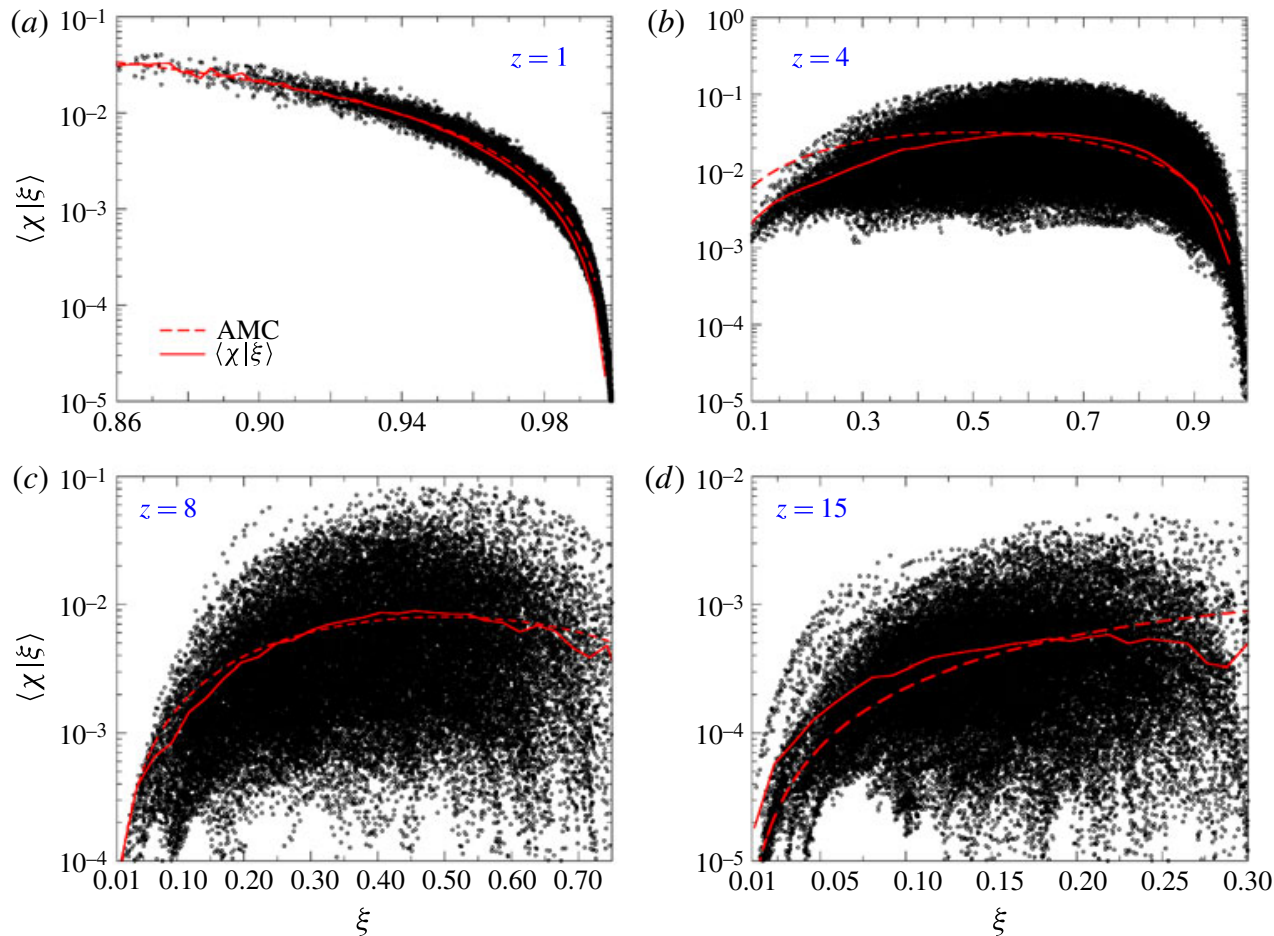

FIGURE 11. (Colour online) $(a-d)$ Scatter plots of conditional scalar dissipation rate $\chi \mid \xi$ along $r=0.5$ at different heights above the nozzle. The conditional mean $\langle\chi \mid \xi\rangle$ (solid line) is compared with the AMC predictions (dashed line).

is often assumed to follow a log-normal distribution (Peters 1983),

$$
P(\ln \chi \mid \xi)=\frac{1}{\sqrt{2 \pi} \sigma_{\ln \chi \mid \xi}} \exp \left\{-\frac{1}{2}\left(\frac{\ln \chi \mid \xi-\mu_{\ln \chi \mid \xi}}{\sigma_{\ln \chi \mid \xi}}\right)^{2}\right\},
$$

where $\mu_{\ln \chi \mid \xi}$ and $\sigma_{\ln \chi \mid \xi}$ are the mean and standard deviation of $\ln \chi \mid \xi$. According to figure 12 , the log-normal distribution at different downstream locations along $r=0.5$ appears to be a reasonable approximation. However, low values of $\chi \mid \xi$ are underestimated, while high values are overestimated, and at the tails, the p.d.f. is closer to being exponential (Lee \& Pope 1995).

\section{Autoignition in a turbulent coflow}

This section presents observations relating to the low-turbulence-intensity simulation, which is closest to that measured experimentally in the CTHC. Qualitatively similar observations concerning the flame behaviour were also made in the high $I_{i}$ case.

\subsection{Global phenomenological observations and flame behaviour}

Phenomenologically, the three-dimensional simulations are consistent with the experimental observations in the CHTC setup for hydrogen (Markides 2005; Markides \& Mastorakos 2005) and other fuels in the 'random spots' regime in terms of the explosive emergence of localized flamelets which then propagate into their unburnt surroundings exhibiting complex interactions. 

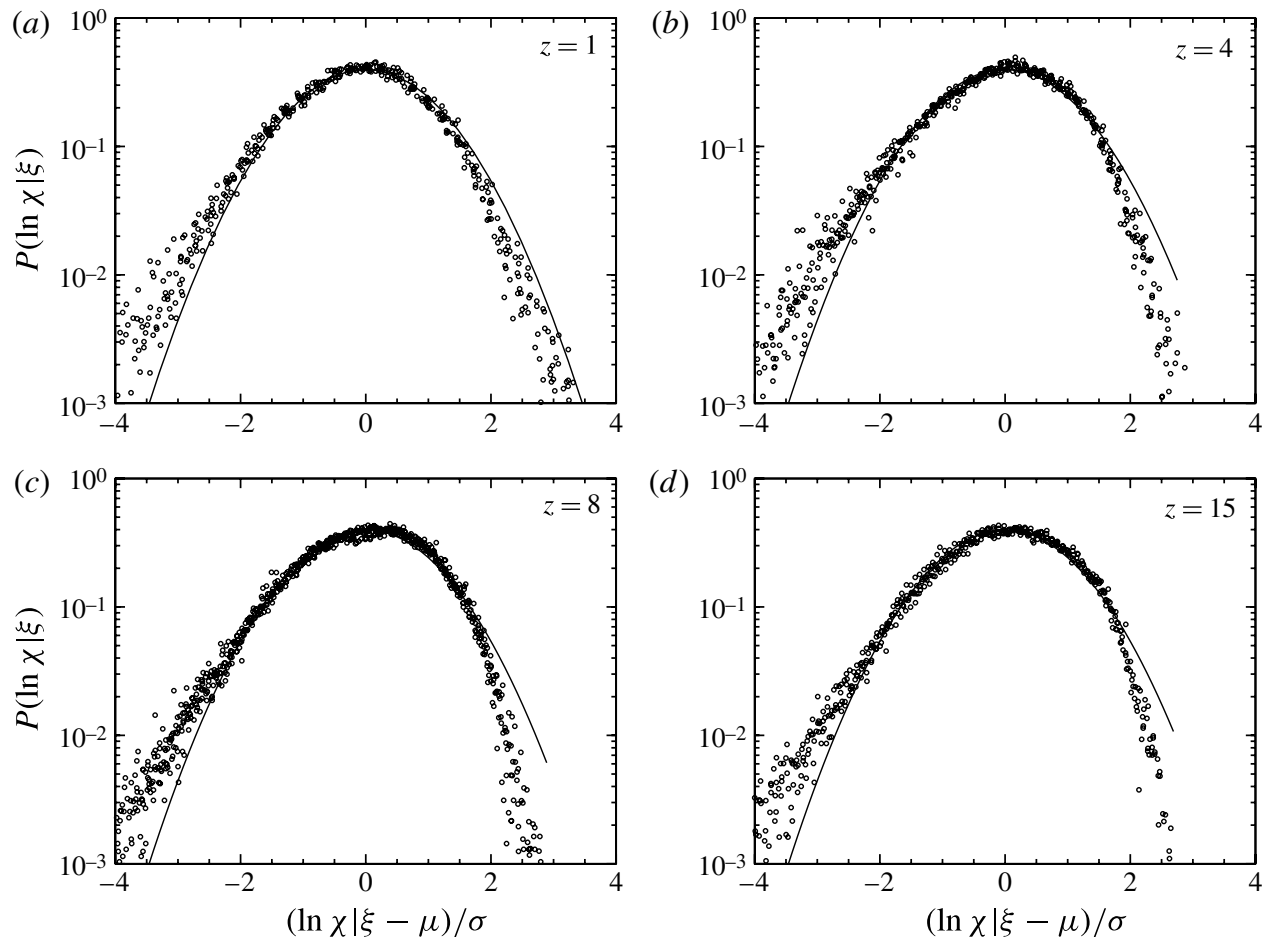

FIGURE 12. $(a-d)$ Evolution of the normalized p.d.f. of $\chi \mid \xi$ for all available $\xi$ with $z$ along $r=0.5$. The solid line indicates log-normal distribution. Here, $\mu$ and $\sigma$ are the mean and standard deviation of $\ln \chi \mid \xi$, respectively.

Two-dimensional simulations (Kerkemeier 2010) reproduced the experimentally observed regimes (table 3). For mean velocities $U_{f}=U_{a}=26 \mathrm{~m} \mathrm{~s}^{-1}$, turbulence intensity $I_{i}=0.15$, fuel composition $Y_{H_{2}}=0.14$ and fuel temperature $T_{f}=855 \mathrm{~K}$, 'no ignition' was observed within the domain at air temperature $T_{a}=930 \mathrm{~K}$. At the highest investigated $T_{a}=1080 \mathrm{~K}$, flame flashback eventually led to an anchored flame. In between these two regimes, 'random spots' were observed for $T_{a}=945$ and $955 \mathrm{~K}$, and a 'lifted flame' with a mean lift-off height of $9 \mathrm{~mm}$ was obtained at $T_{a}=985 \mathrm{~K}$. In the latter case, the simulations clearly showed the dominant effect of the $\mathrm{HO}_{2}$ radical in the stabilization of the flame (Kerkemeier 2010). Instantaneous images of the logarithm of the heat release rate exemplifying the three strongly reacting behaviours are shown in figure 13 .

In terms of mixing, the two-dimensional simulations showed that the stoichiometric mixture fraction isoline extends over the whole domain, in contrast to the threedimensional mixing behaviour ( $\$ 4.2)$. In the random-spot regime, this enabled a flame to hover farther downstream from the AISs. Despite the differences with respect to the turbulent flow and mixing fields, in addition to capturing all of the experimentally observed regimes and the effect of increasing coflow temperature, the two-dimensional simulations were in reasonable agreement with the experiment in terms of the mean height of the AISs.

The three-dimensional simulations focus on the random spots autoignition regime for the conditions outlined in tables 1 and 2 . Volume-renderings of the 
(a)

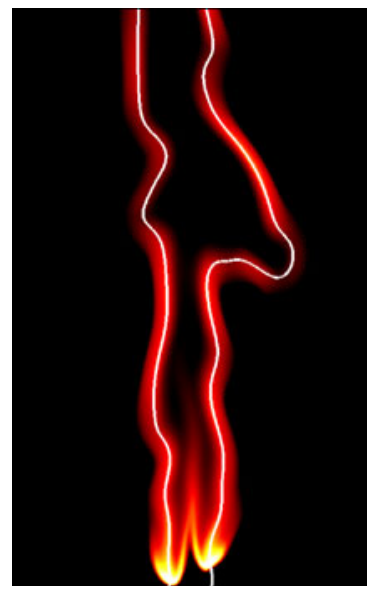

(b)

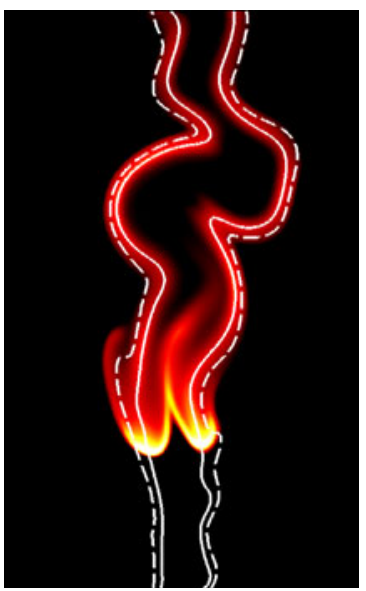

$(c)$

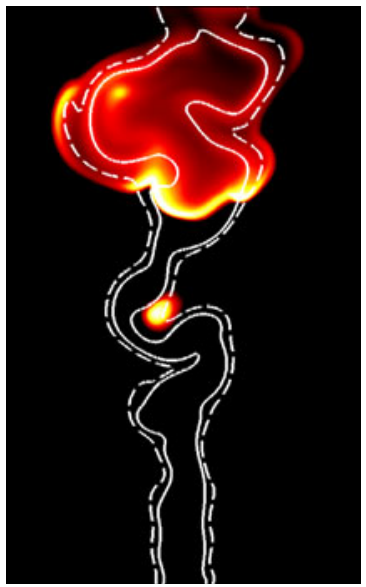

FIGURE 13. (Colour online) Instantaneous images of the logarithm of the heat release rate for the observed regimes in the two-dimensional simulations: $(a)$ anchored flame $\left(T_{a}=1080 \mathrm{~K}\right),(b)$ lifted flame $\left(T_{a}=985 \mathrm{~K}\right)$ and $(c)$ random spots $\left(T_{a}=945 \mathrm{~K}\right)$. The dashed and solid white lines marks the instantaneous most reactive mixture fraction and the stoichiometric mixture fraction isolines, respectively.

Air temperature $(\mathrm{K})$

930

945

955

985

1080
Autoignition regime

No ignition

Random spots

Random spots

Lifted flame

Flashback/anchored flame

TABLE 3. Observed autoignition regimes in two-dimensional DNS as a function of $T_{a}$. In all simulations: $U_{f}=U_{a}=U=26 \mathrm{~m} \mathrm{~s}^{-1}, I_{i}=0.15, Y_{H_{2}}=0.14$ and $T_{f}=855 \mathrm{~K}$.

$\mathrm{OH}$ production rate $\dot{\omega}_{O H}$ were found to produce images that are very similar to the $\mathrm{OH}$ chemiluminescence images obtained experimentally in the CTHC (Markides 2005; Markides \& Mastorakos 2008a, 2011). In figure 14(a), a typical instantaneous realization of $\dot{\omega}_{O H}$ from the simulations is compared with a typical instantaneous (short-exposure) chemiluminescence snapshot from the experiments. Feature similarities are also revealed in figure $14(b, c)$ between contour plots of timeaveraged $\left\langle Y_{\mathrm{OH}}\right\rangle$ and $\left\langle\dot{\omega}_{\mathrm{OH}}\right\rangle$ and experimental time-averaged $\mathrm{OH}$ chemiluminescence images and long-exposure direct photography, respectively. It should be noted though that the experimental images are for acetylene and are provided only to show qualitatively the experimentally observed spots in the same scale as in the simulations. The image quality for hydrogen suffered from very low emitted levels and the noise introduced by the image intensifier. The long-exposure images illustrate the existence of an 'autoignition region', whose axial extent with respect to the injector is characterized by the length $L_{i g n}$. This region can be quantified in a number of ways, as discussed in more detail in $\$ 5.4$.

The series of volume renderings of $\dot{\omega}_{O H}$ in figure 15 reveal some details of typical flame behaviour. The image sequence starts shortly after the appearance of a localized 
(a)

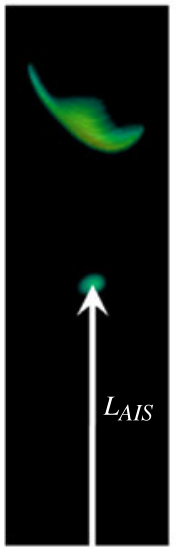

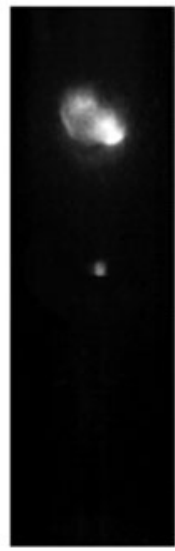

(b)

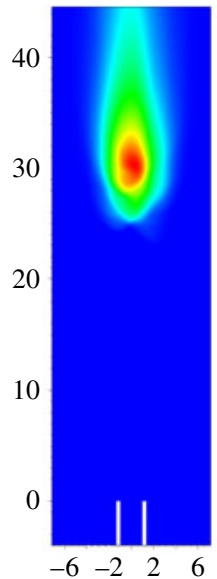

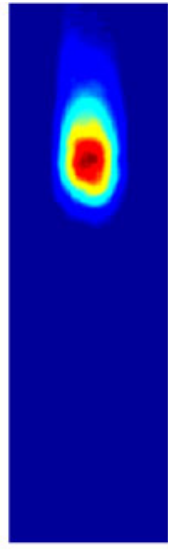

(c)

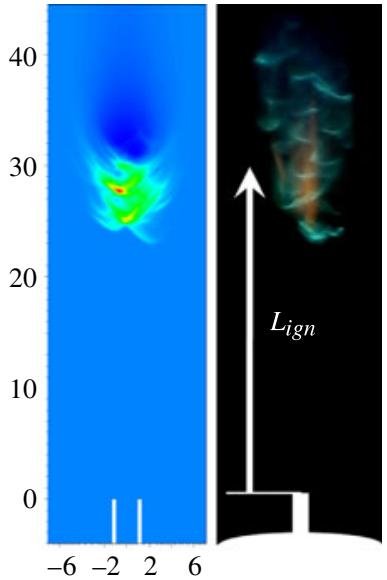

FIGURE 14. (Colour online) (a) Instantaneous images of volume-rendered $\mathrm{OH}$ production rate $\dot{\omega}_{O H}$ from the simulations showing the definition of $L_{A I S}$ with the injector at the bottom of the image (left) and experimental short-exposure $\mathrm{OH}$ chemiluminescence (right); (b) contour plots of time-averaged/-integrated images of $\left\langle Y_{O H}\right\rangle$ from the simulations (right) and experimental $\mathrm{OH}$ chemiluminescence for hydrogen (left); (c) contour plot of $\left\langle\dot{\omega}_{\mathrm{OH}}\right\rangle$ from the simulations (left) and experimental long-exposure photograph of acetylene autoignition from Markides (2005) (right).

Location

$t_{1}$

$n_{\text {AIS }}$

$f_{A I S}=n_{A I S} / t_{T}$

$\min \left\{L_{A I S}\right\}$

$\tau_{A I S}=\left\langle L_{A I S}\right\rangle / U$

$\tau_{A I S} / \tau_{t}$

$\max \left\{L_{A I S}\right\}$

$\left\langle L_{A I S}\right\rangle$

$\sigma_{L, A I S}$

$\sigma_{L, A I S} /\left\langle L_{A I S}\right\rangle$

$\min \left\{X_{A I S}\right\}$

$\max \left\{X_{A I S}\right\}$

$\left\langle X_{A I S}\right\rangle$

$\sigma_{X, A I S}$
Description

Appearance of first AIS

Number of AISs during $t_{T}$

AIS frequency

Minimum axial AIS length

Mean residence time until autoignition

Eddy turnovers until autoignition

Maximum recorded $L_{i g n}$ during simulation

Mean $L_{i g n}$ from simulation

Standard deviation of $L_{i g n}$ from simulation

Standard deviation of $L_{i g n}$ divided by its mean

Minimum radial AIS length $\left(X_{i g n}\right)$

Maximum recorded $X_{i g n}$ during simulation Mean $X_{i g n}$ from simulation

Standard deviation of $X_{i g n}$ from simulation

$\begin{array}{cc}I_{i}=0.15 & I_{i}=0.25 \\ 46.6 & 47.9 \\ 23 & 24 \\ 2.3 \mathrm{kHz} & 2.4 \mathrm{kHz} \\ 22.2 & 22.4 \\ 1.11 \mathrm{~ms} & 1.19 \mathrm{~ms} \\ 1.1 & 1.9 \\ 28.7 & 31.6 \\ 25.6( \pm 1 \%) & 27.5( \pm 1 \%) \\ 1.6( \pm 15 \%) & 2.0( \pm 15 \%) \\ 0.06 & 0.07 \\ & \\ 0.8 & 0.5 \\ 3.2 & 4.7 \\ 2.1( \pm 7 \%) & 2.7( \pm 8 \%) \\ 0.7( \pm 15 \%) & 1.1( \pm 15 \%)\end{array}$

TABLE 4. Spatial location of AISs in (low) $I_{i}=0.15$ and (high) $I_{i}=0.25$ cases. Standard errors for the mean and standard deviation are shown in parenthesis, assuming a normal distribution. All values are normalized by $r_{i}$.

AIS at about one-third of the image height, upstream of an existing larger 'postignition flamelet' (PIF). In the first row of images, the partially premixed flame initiated from the newly formed AIS propagates outwards, while the PIF remains situated approximately one-third of the image height farther downstream. A second AIS appears at $t=186.45$, and the propagating PIF fronts from the two AIS meet and interact while propagating towards the PIF lingering above them. At $t=187.65$, the 

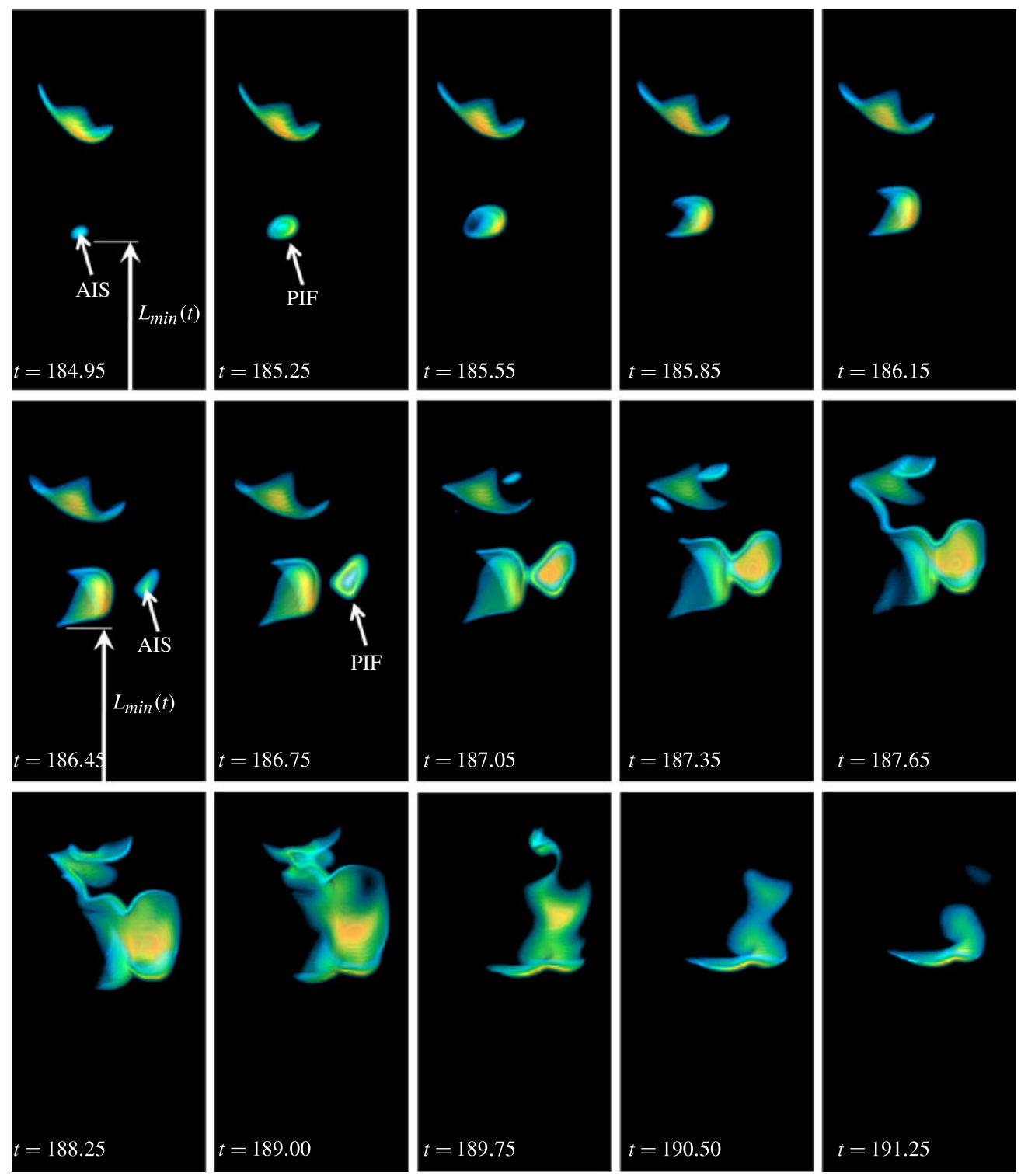

FIGURE 15. (Colour online) Zoomed-in sequence of volume-rendered images of $\mathrm{OH}$ production rate $\dot{\omega}_{\mathrm{OH}}$ showing AIS formation and PIF propagation, as well as PIF interactions. Also showing the definition of $L_{\min }(t)$.

three PIFs merge into a single flamelet. Subsequently, this flamelet slowly extinguishes, while at the most upstream point the flamelet gives rise to a region of stronger reaction $(t=190.50)$. Eventually, only the upstream front remains, which is advected slowly downstream by the flow with an absolute axial velocity of $u_{P I F} \approx 0.3$. The interactions can either act to sustain them for some time or to extinguish them, reflecting closely the experimental observations (Markides 2005; Markides \& Mastorakos 2008a).

The temporal evolution of the integral of the heat release rate $i H R R$ over the entire domain (rendered non-dimensional by the density, heat capacity and temperature 

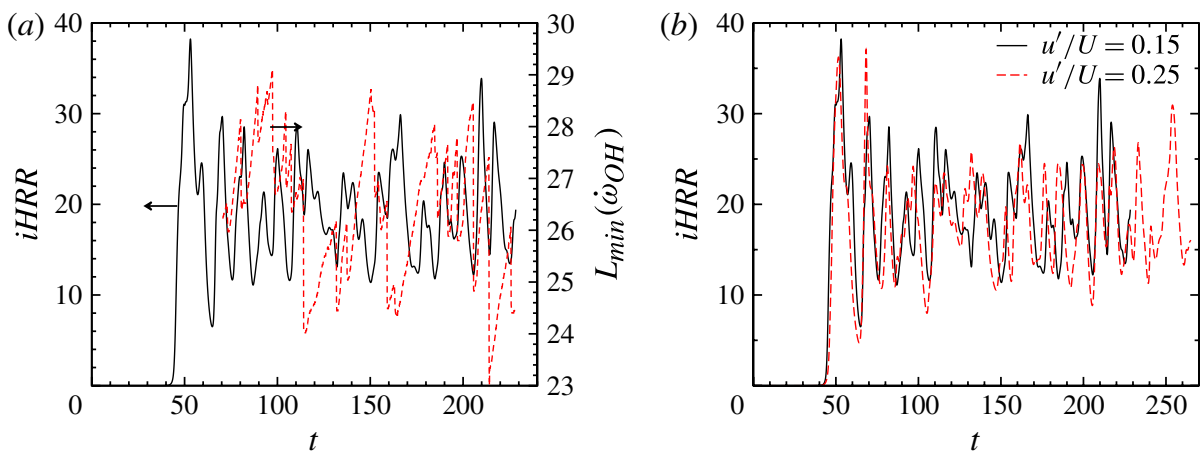

FIgURE 16. (Colour online) Temporal evolution of $(a)$ the dimensionless volume-integrated heat release rate in the whole domain $i H R R$ (solid line) and minimum axial length of $\mathrm{OH}$ production rate $L_{\min }$ (dashed line) for the low $I_{i}=0.15$ case and (b) the dimensionless volumeintegrated heat release rate in the whole domain $i H R R$ for the low $I_{i}=0.15$ case (solid line) and high $I_{i}=0.25$ (dashed line).

of the fuel jet) reported in figure $16(a)$ shows that autoignition in this case first occurs after a delay of $t_{1}=46.6$ from the introduction of hydrogen into the fuel stream. Subsequently, the $i H R R$ signal contains peaks and troughs appearing at random intervals. The peaks are associated with the appearance of AIS, such as those shown in figure 15. The iHRR plot is overlaid with the simultaneous value of $L_{\text {min }}$, the minimum axial distance from the injector nozzle to the most upstream location of regions of high $\mathrm{OH}$ production rate (dashed line). The latter is obtained by processing each computed image to identify the minimum axial distance from the nozzle having pixel intensity above a threshold of $3 \%$ of the maximum value (see also figure 15). Careful inspection reveals periods of gradual increase in $L_{\min }$ corresponding to the slow propagation of PIF downstream, interrupted by occasional sharp upstream shifts. The sudden upstream shifts are accompanied by a sharp increase in $i H R R$ and mark the emergence of a new AIS at an upstream location. The saw-tooth-shaped profile of the heat release rate and of $L_{\text {min }}$ are similar to the measured temporal evolution of the lift-off height of a diesel jet in a high-temperature, high-pressure spherical bomb which was attributed to consecutive autoignition events (Yoo et al. 2011).

Preliminary investigations of the balance of the reactive and diffusive terms within a spot indicates that the kernels ignite homogeneously, and quickly form propagating fronts, but the analysis of the autoignition kernel structure and the dynamics and interaction of the PIFs will be the focus of a future investigation.

\subsection{AIS frequency}

A total of 23 (low $I_{i}$ run) and 24 (high $I_{i}$ run) independent AISs were recorded during the simulation time after the introduction of hydrogen in the fuel stream $t_{T}$, resulting in average autoignition event frequencies of $f_{\text {ign }}=2.3-2.4 \mathrm{kHz}$. Table 4 summarizes these results, while figure 16(b) compares the $i H R R$ histories of the lowand high-turbulence-intensity cases. These frequencies are in good agreement with the experimental value of approximately $2 \mathrm{kHz}$ in similar 'fast spotting' conditions when autoignition was observed at short distances from the injector (Markides 2005; Markides \& Mastorakos 2011).

With respect to the autoignition length $L_{i g n}$ in table 4 , it should be noted that: (i) $L_{A I S}$ (as in figure 14) is a measure of the axial length from the injector nozzle to 
the point of the emergence of an independent AIS, as judged by visual inspection of the $\mathrm{OH}$ production rate, such that there are only as many samples of $L_{A I S}$ generated as there are AIS realizations; whereas (ii) the previously employed $L_{\text {min }}(t)$ (figures 15 and $16 a$ ) is the continuous measure in each successive image of the axial length from the injector nozzle to the most upstream regions of high $\dot{\omega}_{O H}$ due to an AIS or a PIF. The two measures may occasionally coincide at some instances such as that shown in figure 14(a), but will generally deviate due to flame propagation following localized autoignition.

It is interesting to note that although the first realization of thermal runaway associated with an AIS in the high $I_{i}$ case appears slightly delayed relative to the corresponding first AIS in the low $I_{i}$ case (figure 16b), the two cases give rise to qualitatively similar $i H R R$ signatures and frequencies. This is in agreement with the experimental observation that in the cases where $\left\langle L_{\text {min }}\right\rangle$ was kept short by increasing the air temperature, $f_{i g n}$ was not significantly altered when $U_{a}$ (and, hence, also the turbulent velocity fluctuations $u^{\prime}$ ) were increased by a factor of 1.6 (Markides 2005; Markides \& Mastorakos 2008a). Nevertheless, the first emergence of an AIS is delayed by 1.3 time units at higher $I_{i}$, indicating a delaying effect of higher $u^{\prime}$ on the pre-ignition chemistry.

As a final comment, it can be seen that $\min \left\{L_{A I S}\right\}$ is affected only minimally by the change in $I_{i}$, whereas $\left\langle L_{A I S}\right\rangle$ is longer by $7 \%$ when $I_{i}$ is increased. We will return to the spatial appearance of AIS in the domain in $\S 5.4$.

\subsection{Appearance of autoignition and post-ignition flames in mixture fraction space}

Scatter plots of temperature $T$, mass fractions $\mathrm{HO}_{2}$ and $\mathrm{OH}$, and thermal scalar dissipation rate $\chi$ (first to fourth row) against the local mixture fraction $\xi$ were compiled over time and over horizontal planes normal to the flow axis at $z=1,12,23$ and 29 (first to fourth column) (figure 17, low $I_{i}$ case). As indicated by the thermal runaway in the scatter plots of temperature and $Y_{O H}$, the AISs appear at $z \approx 22$. The corresponding conditional means are shown in some plots. Further downstream $(z>23)$, the scatter plots become contaminated with post-ignition combustion and PIF interactions.

In line with the results presented in $\$ 4.2 .2$, the scatter plots reveal high scalar dissipation rates close to the nozzle which decays quickly downstream. Keeping in mind that the reported values are non-dimensionalized with respect to $\chi_{\text {ref }}=1 / t_{\text {ref }}=$ $23111.1 \mathrm{~s}^{-1}$, it can be seen that close to the nozzle $\chi$ can reach, albeit not very often, values up to $3200 \mathrm{~s}^{-1}$, which are higher than the critical scalar dissipation above which autoignition is precluded for the conditions under consideration $\left(\chi_{\text {crit }} \approx 2650 \mathrm{~s}^{-1}\right.$ evaluated by two-dimensional laminar counterflow simulations). The conditional average $\langle\chi \mid \xi\rangle$ can fail to describe adequately the range of observed values of $\chi \mid \xi$, with considerable fluctuations $\chi^{\prime \prime} \mid \xi$ present around the conditional mean, especially at long $z$ (see for example the scatter plot at $z=23$ in figure 17). These deviations can affect the eventual location of autoignition through their effect on the pre-ignition chemistry.

The conditional mean of $Y_{\mathrm{HO}_{2}}$ is indicated at $z=1$ and $z=23$ in order to clarify how the local $\xi_{M R}$ was defined. The $\mathrm{HO}_{2}$ radical starts forming very close to the nozzle exit, and $\mathrm{Y}_{\mathrm{HO}_{2}}$ increases strongly with height in the pre-ignition region. Since $\mathrm{OH}$ is not produced in significant amounts, the local most reactive mixture fraction in the pre-ignition region $\xi_{M R}$ is defined by identifying the peak in the conditional mean of $\left\langle Y_{\mathrm{HO}_{2}} \mid \xi\right\rangle(z)$. The dependence of the local $\xi_{M R}$ on $\left\langle\chi \mid \xi_{M R}\right\rangle$ (figure 18) reveals that close to the nozzle pre-ignition chemistry is taking place at conditions richer 

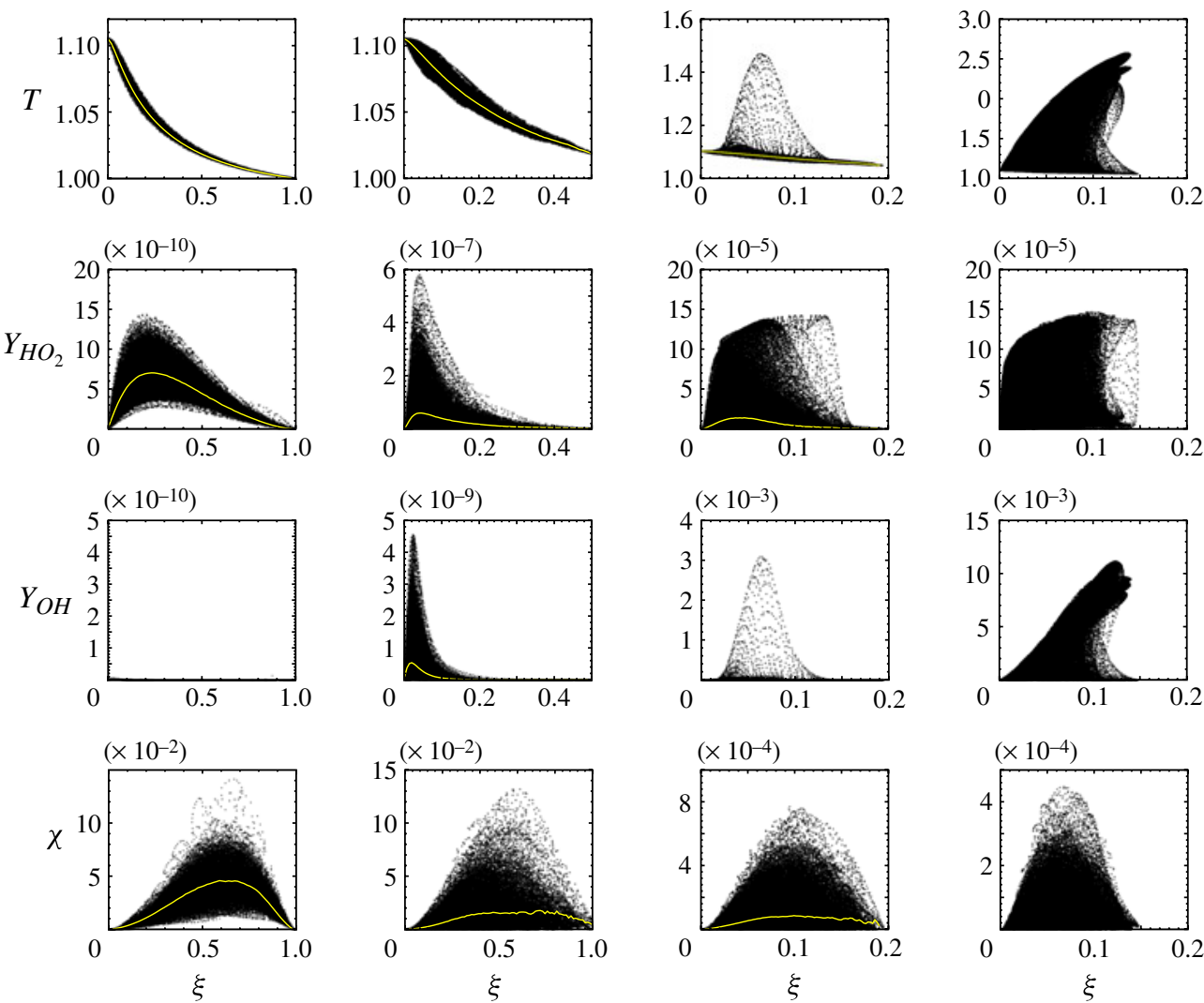

FIgURE 17. (Colour online) Scatter plots of temperature $T$ (first row), the mass fractions $Y_{\mathrm{HO}_{2}}$ (second row) and $Y_{\mathrm{OH}}$ (third row), and scalar dissipation rate $\chi$ (fourth row) as a function of the mixture fraction $\xi$ on planes normal to the axial direction at $z=1,12,23,29$ (first to last column). Superimposed in some plots are the corresponding conditional means (solid lines).

than stoichiometric $\left(\xi_{M R} \approx 0.27\right)$, but $\xi_{M R}$ decays quickly and by $z \approx 6$ reaches the homogeneous value of $\xi_{M R, 0}=0.035$. Variation of the instantaneous $\xi_{M R}$ with time was also reported by Sreedhara \& Lakshmisha (2002) in their three-dimensional DNS when a four-step scheme was used to describe $n$-heptane kinetics; for a single-step kinetic model $\xi_{M R}$ was constant. Thus, as with previous numerical studies on turbulent autoignition, autoignition is observed eventually in regions where $\chi$ is low and at a value of $\xi_{M R}$ that is close to that estimated from homogeneous calculations $\left(\xi_{M R, 0}\right)$. Even though initially $\chi_{M R}$ may be high (more than an order of magnitude higher than $\chi_{M R, 0}$ here), during the autoignition delay time turbulence mixes the reactants and $\chi_{M R}$ decays, and $\xi_{M R}$ approaches the value of $\xi_{M R, 0}$.

The scatter plots at $z$ higher up in the domain show the evolution to a burning state (last two columns of figure 17), and exhibit similarities with the scatter plots reported by Cabra et al. $(2002,2005)$. The temperature peak shifts progressively to richer $\xi$ and reaction fronts appear in very lean and very rich mixtures, showing that PIF can exist in such mixtures following autoignition at $\xi_{M R}$. 


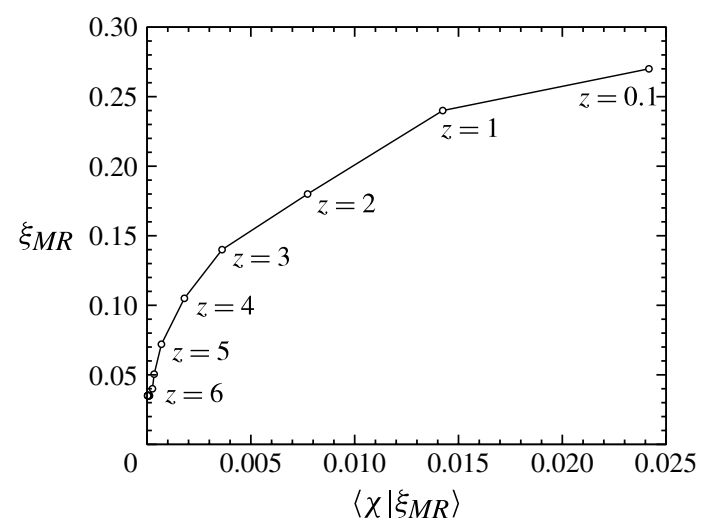

FIGURE 18. Most reactive mixture fraction $\xi_{M R}$ as a function of the conditional mean of $\chi$ at $\xi_{M R}$.

\subsection{Mixing and autoignition location}

Statistics of the axial and radial locations of the observed AISs are detailed in table 4. These were compiled from the total of 23 and 24 independent AISs generated over the available simulation time $t_{T}$ in the low and high $I_{i}$ simulations, respectively.

\subsubsection{Autoignition location statistics}

In figure $7(c)$, the locations of all recorded autoignition events in the low $I_{i}$ simulation are superimposed on the isocontours of the mean mixture fraction $\langle\xi\rangle$ and of $\langle\xi\rangle=\xi_{s t}$ and $\langle\xi\rangle=0.05$, a value representative of the most reactive mixture fraction $\xi_{M R}$. As discussed in $\S 4.2,\langle\xi\rangle$ decays along the centreline as $z^{-1.5}$ sufficiently far away from the nozzle, dropping below the stoichiometric value $\xi_{\text {st }}=0.17$ at $z \approx 15$ (figure 8). The appearance of the AISs at locations where the local composition is on average much leaner than stoichiometric has a considerable effect on the observed combustion behaviour. It can be expected that external perturbations resulting in the appearance of spots within the $\xi_{s t}$ isosurface can lead to the stabilization of a lifted flame, which will be supported by autoignition ahead of the flame base. Furthermore, the absolute axial advection velocities of the PIF were of the order of $u_{P I F} \approx 0.3$ (approximately $7.8 \mathrm{~m} \mathrm{~s}^{-1}$ ). In the CHTC experiments (Markides \& Mastorakos 2008a), the measured absolute flame velocities were in the range $6.5-8.5 \mathrm{~m} \mathrm{~s}^{-1}$ for hydrogen with $U_{a}=25 \mathrm{~m} \mathrm{~s}^{-1}$ and $T_{a}=950 \mathrm{~K}$.

The statistics of the axial and radial locations of the AISs including relative standard error estimates for the mean and standard deviation of the different lengths are summarized in table 4; a normal distribution is assumed. The assumption is reasonable as it has been established in the experiments (Markides 2005; Markides et al. 2007) that $L_{A I S}$ was approximately normally distributed. In the low $I_{i}$ simulation, the most upstream AIS is observed at $\min \left\{L_{A I S}\right\}=22.4$. The average axial and radial autoignition locations relative to the injector are $\left\langle L_{A I S}\right\rangle=25.6$ and $\left\langle X_{A I S}\right\rangle=2.1$, respectively. From the mean axial autoignition length $\left\langle L_{A I S}\right\rangle$, it is possible to define a 'mean residence time until autoignition' $\tau_{A I S}$ based on a characteristic advection velocity. Using the bulk velocity $U=26 \mathrm{~m} \mathrm{~s}^{-1}$, we obtain a $\tau_{A I S}=1.11 \mathrm{~ms}$, which corresponds to 1.1 eddy turnover times and is approximately equal to the homogeneous autoignition delay time $\tau_{i g n, 0}$. The standard deviations of $L_{A I S}$ and $X_{A I S}$ are 1.6 and 0.7, and hence the statistical standard errors $\epsilon=\sigma / \sqrt{n}$ in $\left\langle L_{A I S}\right\rangle$ and $\left\langle X_{A I S}\right\rangle$ 


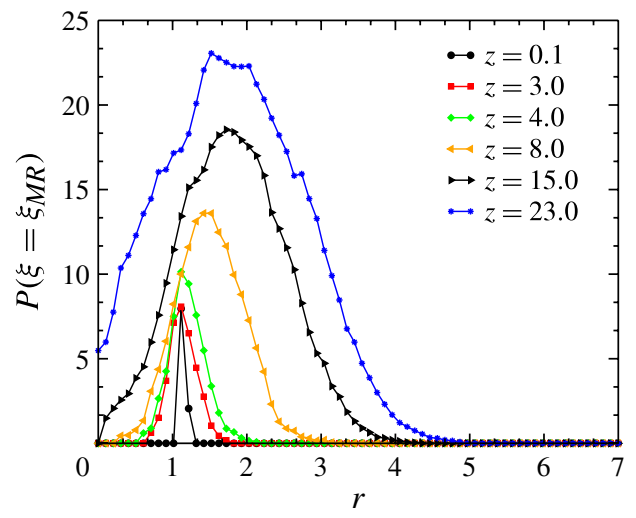

FIGURE 19. (Colour online) Value of the p.d.f. of $\xi=\xi_{M R}$ as a function of radius at different axial locations $\left(I_{i}=0.15\right.$ case). Note that each line is not a p.d.f.; it is formed by joining points each one of which comes from compiling a p.d.f. over time at a particular radial and axial location $(r, z)$, and identifying the value of the p.d.f. at the MR mixture $\xi=\xi_{M R}$.

are 0.33 and 0.14 , respectively. Experimental results for the same mixture and velocities but with $T_{a} \approx 955 \mathrm{~K}$ showed $L_{\text {min }} \approx 22$ and $\left\langle L_{\text {min }}\right\rangle \approx 25$.

The AISs tend to be located off-axis and the average radial autoignition location $\left\langle X_{A I S}\right\rangle$ matches closely the radial location of the $\langle\xi\rangle=\xi_{M R}$ isosurface at the height corresponding to $z=\left\langle L_{A I S}\right\rangle$. Since it was established in $\S 5.3$ that all AISs appear at locations where instantaneously $\xi \approx \xi_{M R, 0}$, it is interesting to probe the probability of the emergence of a mixture with the right composition for autoignition $\xi_{M R}$ and how this varies in the domain. Using the mixture fraction p.d.f. $P(\xi)$ extracted from the simulations, the radial dependence of $P\left(\xi=\xi_{M R} ; r, z\right)$ at different heights from the injector is plotted in figure 19. The peak matches closely the radial location of $\langle\xi\rangle=\xi_{M R}$ at $z=\left\langle L_{A I S}\right\rangle$ and agrees well with the mean radial autoignition location $\left\langle X_{A I S}\right\rangle$, showing that autoignition arises at radial locations where the probability of finding pockets with the optimal composition is the highest.

\subsubsection{Autoignition location randomness}

Despite the small number of spots, considerable scatter exists in the AIS appearance (see figure $7 c$ and table 4 ). In the low $I_{i}$ simulation, AISs were observed over the range of axial and radial locations spanning $22.2<z<28.7$ and $0.8<r<3.2$, with a corresponding standard deviation in $L_{A I S}$ and $X_{A I S}$ of 1.6 and 0.7 . We note that the axial r.m.s. of the spreading is by a factor of 2.3 greater that the radial, and the axial spreading amounts to $6 \%$ of $\left\langle L_{A I S}\right\rangle$.

The radial profiles of $P\left(\xi=\xi_{M R} ; r, z\right)$ (figure 19) correlate with the off-axis regions within which AISs appear. For $z \leqslant\left\langle L_{A I S}\right\rangle$, there is a low probability of finding pockets of $\xi_{M R}$ mixture at $r>4$, consistent with the observation that no AISs were observed there during the simulation time $t_{T}$. In addition, although from about $z>18$ regions of $\xi_{M R}$ appear at the centreline, the probability of this occurring is lower by a factor of 3-5 than that found in the region $1<r<3$ where the AISs actually appear.

As expected from the discussion in $\$ 4.2 .2$, the conditional scalar dissipation rate at most reactive conditions $\chi_{M R}=\chi \mid \xi_{M R}$ decayed quickly. Radial profiles of $\left\langle\chi_{M R}\right\rangle$ (figure 20) reveal that this quantity is consistently higher closer to the centreline relative to the edge of the plume as this spreads downstream. 


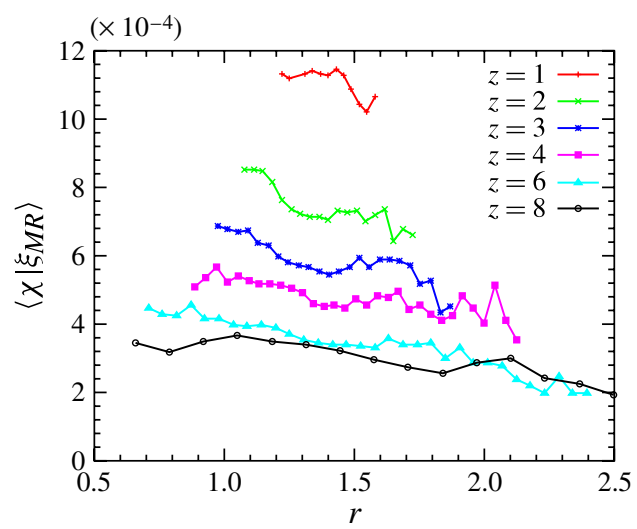

FIGURE 20. (Colour online) Radial profiles of the mean conditional scalar dissipation rate $\left\langle\chi_{M R}\right\rangle=\left\langle\chi \mid \xi_{M R}\right\rangle$ at different axial locations for the low-turbulence-intensity case.

\subsubsection{Effect of scalar mixing on autoignition}

Mixing effects on the chemical processes leading to autoignition can be understood by the history of $\chi_{M R}$, the conditional scalar dissipation rate at the most reactive mixture fraction. In order to observe this history individual kernels of high $\mathrm{HO}_{2}$ concentration were detected to track their trajectories. Different mass fraction isovalues ranging from $10^{-6}$ to $10^{-4}$ were used to define the kernel, all leading to the same observations. Well-defined iso- $\mathrm{HO}_{2}$ concentration kernels could be identified above $z \approx 10$, all of which underwent thermal runaway and resulted in successful autoignition events. However, independent of the isovalue chosen, these kernels could not be traced towards the region of high scalar dissipation rate close to the fuel nozzle as their concentration dropped to very low levels.

Furthermore, their trajectories were found to deviate strongly from the path lines followed by non-reactive particles initialized within these kernels. The inspection of the individual histories of these radical pockets could not explain the spreading in the autoignition location, since the differences in the scalar dissipation rate experienced by the kernels was too small to account for the variation in the autoignition length. This suggests that the autoignition lengths of the individual AIS locations from the fluctuating scalar dissipation rate and velocity close to the fuel nozzle (i.e. $z \lesssim 10$ ), where it was not possible to track the kernels. The considerably higher scalar dissipation rate in this region is demonstrated in figures 18 and $21(a)$, which shows that the conditional scalar dissipation rate $\left\langle\chi \mid \xi_{M R}\right\rangle$ calculated at $z=10$ is two orders of magnitude higher than that found in the region $z<1$ and one order of magnitude higher than that in the region $2<z<5$.

In the absence of inhomogeneities, we would have expected the fluid particles to autoignite at the homogeneous ignition delay time $\tau_{i g n, 0}=1.1 \mathrm{~ms}$. In an attempt to transform the residence time into a spatial length that can be compared with the AIS results from the three-dimensional simulations and to quantify any mixing effects, a characteristic velocity of the pre-ignition kernels was employed, which was computed as the axial component $w$ of the propagation velocity of points on the $\xi_{M R}$ isosurface 
(a)

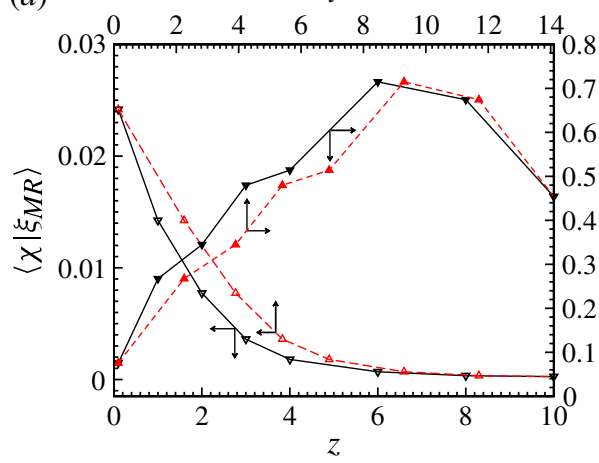

(b)

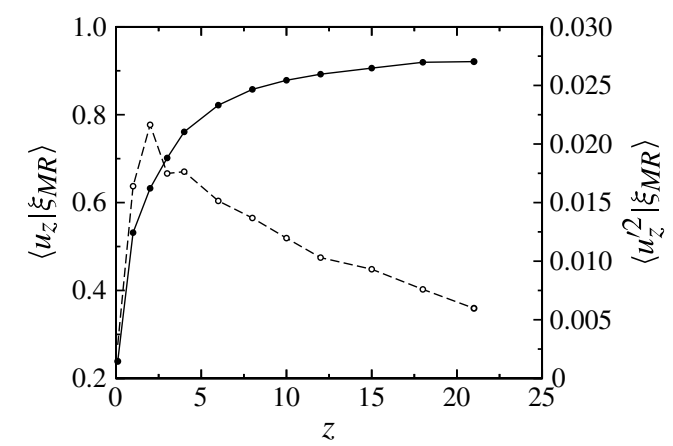

FIgURE 21. (Colour online) (a) Mean scalar dissipation rate conditioned on $\xi_{M R}(\nabla)$ and r.m.s. over the mean scalar dissipation rate conditioned on $\xi_{M R}(\boldsymbol{\nabla})$ versus axial location $z$ (solid line) and time $t$ (dashed line). (b) Mean (solid line with filled circles) and variance (dashed line with open circles) of velocity conditioned on MR $u \mid \xi_{M R}$ against axial location $z$ for the low $I_{i}$ turbulence intensity case.

as (Gibson 1968)

$$
w_{K E R}(z, t)=\left\langle w\left|\xi_{M R}-\frac{\mathrm{D} \xi}{\mathrm{D} t}\right|_{\xi_{M R}}|\nabla \xi|^{-1}\left(\underline{n}_{\xi_{M R}} \cdot \underline{k}\right)\right\rangle
$$

i.e. the local conditional axial fluid velocity corrected by the diffusion velocity in the direction of $\underline{n}_{\xi_{M R}}$, the local unit normal vector to the $\xi_{M R}$ isosurface; $\underline{k}$ is the unit vector in the axial direction. The averaging $\langle\cdots\rangle$ is performed along the instantaneous $\xi_{M R}$ on planes at a height $z$ above the nozzle.

The Lagrangian time $\tau$ and the height of the AIS $z$ can then be related by

$$
\tau(z, t)=\int_{0}^{z} \frac{\mathrm{d} z}{w_{K E R}(z, t)} ; \quad z(\tau)=\int_{0}^{\tau} w_{K E R}(z(t)) \mathrm{d} t .
$$

This Lagrangian tracking procedure takes into account advection and diffusion of points with a concentration $\xi_{M R}$ with velocity $w_{K E R}(z, t)$. It is based on the observation that the pre-ignition chemistry is constrained on mixtures around $\xi_{M R}$ and the fact that the path of autoignition precursors is different from that of a non-reactive fluid particle. The calculation takes into account the low-velocity regions in the injector boundary layers and in the injector wake. It is noted that in the wake region $u_{M R}=\left\langle u \mid \xi_{M R}\right\rangle$ is actually low, starting from $\sim 0.24 U$, and increasing quickly to $0.86 U$ by about $z \approx 10=4 d_{i}$ (figure $21 b$ ).

Another way to view $\tau_{i g n, 0}$ is that it reveals the shortest time that we would have expected autoignition to appear had we established the mixing state with $\xi=\xi_{M R, 0}$ infinitely fast. Similarly, integrating $\underline{u}_{K E R}(\underline{r})$ from 0 to $\tau_{\text {ign }, 0}$, a homogeneous autoignition length $L_{i g n, 0}=20.3$ is obtained, revealing the shortest distance from the injector where we would have expected autoignition to appear had we established the state $\xi=\xi_{M R}$ condition infinitely fast. Considering that for all AIS realizations $1.09<L_{A I S} / L_{i g n, 0}<1.41$ and that on average $\left\langle L_{A I S}\right\rangle / L_{i g n, 0}=1.26$, scalar mixing is seen to have a noticeable delaying effect on the autoignition location. It should be pointed out that a fixed value $\xi_{M R}=0.05$ was used in the analysis for the most reactive mixture fraction instead of the axially varying $\xi_{M R}(z)$ (figure 18), since (5.1) assumes a 
fixed isovalue. Since the only significant discrepancy between these two values occurs at short $z<6$, the location of the MR isocontour and, hence, also of the velocity of the MR isocontour $\underline{u}_{M R}$ is not expected to vary appreciably with the choice of $\xi_{M R}$.

\subsubsection{Effect of turbulence intensity level}

A simulation with an enhanced turbulence intensity $\left(I_{i}=0.25\right)$ was performed to investigate the effect of turbulence on autoignition. The statistics of the autoignition location are summarized and compared to their low $I_{i}$ counterparts in table 4 . No change in the mean AIS frequency was observed. This insensitivity to turbulence intensity can be attributed to the extended plateau of the homogeneous autoignition delay time, and the fast decrease of the scalar dissipation rate, which result in the existence of optimal autoignition conditions at some location in the domain (even at different radial locations on approximately the same axial distance from the nozzle). It is also possible that while frequency does not appear to depend on the turbulence intensities considered, it may depend on the integral length scale $l_{I}$ so that a scaling exists with respect to the eddy turnover time $\tau_{t}=l_{I} / u^{\prime}$. Since $l_{I}$ was kept constant in this study, new simulations will be needed to address this point.

For high $I_{i},\left\langle L_{A I S}\right\rangle=27.5$ and $\sigma_{L, A I S}=2.0$, resulting in a statistical convergence error in $\left\langle L_{A I S}\right\rangle$ of $\epsilon=0.43$. The increase in $\left\langle L_{A I S}\right\rangle$ relative to the low $I_{i}$ case is 1.9 (or $7 \%$ ), considerably larger than the statistical uncertainty in $\left\langle L_{A I S}\right\rangle$. In addition, $\tau_{A I S}=1.19 \mathrm{~ms}$ or 1.9 eddy turnover times, compared with $1.11 \mathrm{~ms}$ and 1.1 eddy turnovers from the $I_{i}=0.15$ run. Hence, the enhanced turbulence levels are associated with autoignition that appears further downstream, suggesting a delaying effect of turbulence on autoignition. On the other hand, the minimum autoignition length was minimally affected.

The on-average spatial and temporal delay in autoignition is directly in line with experimental findings in the CHTC, where an increase in the bulk $U=U_{a}=U_{f}$ (and, hence, also $u^{\prime}$ and $\chi$ ) leads to a similar delay in autoignition (Markides 2005). It should be pointed out, however, that the simulations are subtly different from the experiments. Here, the bulk velocity is the same in both low- and high-turbulenceintensity cases, whereas in the experiments the turbulence intensity at the injector was almost constant, but the turbulent fluctuations $u^{\prime}$ were modified by changing the bulk velocity through a turbulence grid upstream of the injector. Thus, the numerical results complement the experimental conclusion that in the presence of strong advection enhanced turbulence acts to spatially and temporally delay autoignition, in contrast to what we would have expected from the three-dimensional autoignition studies of initially spheroidal $n$-heptane packets in hot, stagnant, decaying turbulent air of Sreedhara \& Lakshmisha (2002). They reported that $\tau_{i g n}$ remained unaffected by a decrease of $\tau_{t}$ by up to $50 \%$ in the regime $\tau_{i g n} / \tau_{t} \approx 0.6-1.2$, and that it decreased (i.e. autoignition accelerated) by $10-20 \%$ as they decreased $\tau_{t}$ by $30-50 \%$ in the regime $\tau_{i g n} / \tau_{t} \approx 2.3-3.5$. The corresponding time scale ratios from table 4 are $\tau_{A I S} / \tau_{T}=1.1$ and 1.9 for the low and high $I_{i}$, respectively.

Current understanding suggests that turbulence intensity will affect the pre-ignition chemistry and consequently the emergence of autoignition through its interaction with the scalar field. The delay can be a result of the increase in the conditional scalar dissipation rate at most reactive conditions. To understand the underlying reasons behind the delayed autoignition in the higher-turbulence-intensity simulation, the p.d.f. $P\left(\chi \mid \xi_{M R}\right)$ is examined. Figure 22 shows a representative case of the p.d.f. and the cumulative distribution function (CDF) of $\chi \mid \xi_{M R}$ at $r=1$ and $z=1$; similar observations were made at different radial and axial locations. 


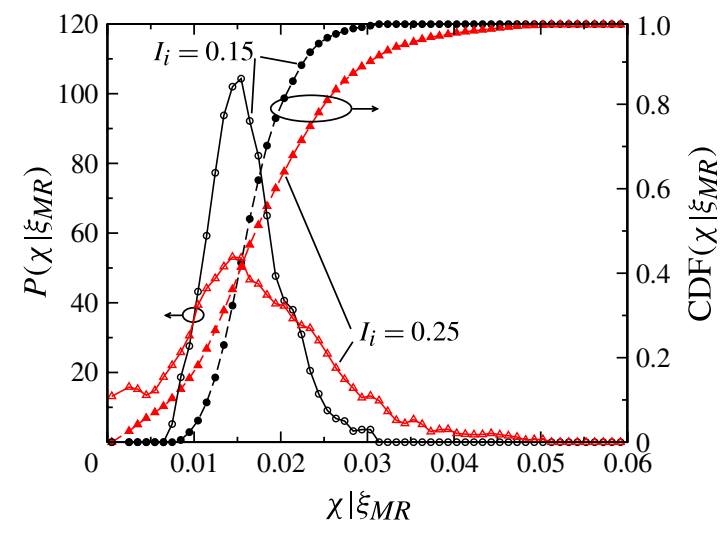

FIgURE 22. (Colour online) Cumulative distribution function (dashed lines with filled symbols) and p.d.f. (solid lines with open symbols) of scalar dissipation rate conditioned on $\xi_{M R}$ for $r=1$ and $z=1$ for $I_{i}=0.15$ (circles) and $I_{i}=0.25$ (triangles).

Enhanced turbulence intensity results in increased p.d.f. values at low and high values of $\chi \mid \xi_{M R}$, while the probability of intermediate values decreased. Indeed, on average the increase in $I_{i}$ leads to an increase in $\left\langle\chi_{M R}\right\rangle$. This offers a reasonable explanation for the delaying effect of turbulence and agrees with the observation that the mean autoignition length increased, while the minimum autoignition length was not significantly affected. The weak dependence of $L_{\min }$ on $\chi \mid \xi_{M R}$ (and hence $I_{i}$ ) is consistent with the explanation provided by Mastorakos et al. (1997a) that somewhere in the turbulent flow the best possible history of $\chi \mid \xi_{M R}$ will emerge.

In the higher $I_{i}$ simulation, autoignition also shifted farther off-axis, with $\left\langle X_{A I S}\right\rangle=$ 2.7. AISs covered the spatial region $22.2<z<31.6$ and $0.5<r<4.7$, resulting in axial and radial standard deviations of $\sigma_{L, A I S}=2.0$ and $\sigma_{X, A I S}=1.1$. Both the axial and radial spreading in the AIS location exhibit an increase relative to the $I_{i}=0.15$ case. The increased axial spread in the autoignition locations was found to be consistent with the increased fluctuations in $\chi \mid \xi_{M R}$ (i.e. broader p.d.f. of $\chi \mid \xi_{M R}$ ), while the radial spread was linked to the increased turbulent dispersion of the fuel plume (i.e. likelihood of $\xi_{M R}$ increased for low and high values of $r$ ).

\section{Conclusions}

The autoignition of a nitrogen-diluted hydrogen plume in a turbulent coflowing stream of high-temperature air was simulated numerically in a laboratory-scale set-up in order to obtain the complete state of the system which was not available from the experiments in a similar configuration (Markides 2005). The analysis of the flow and mixing fields revealed that far enough from the injector the flow field becomes nearly uniform and the fast mixing of fuel and oxidizer results in very low scalar dissipation rate $\chi$ sufficiently far downstream, the mixing pattern being between that of a plume and an axisymmetric jet. The p.d.f. of the mixture fraction $\xi_{M R}$ was found to be in excellent agreement with a posteriori evaluated two-parameter beta p.d.f., while the p.d.f. of the conditional scalar dissipation rate p.d.f. $(\chi \mid \xi)$ follows a log-normal distribution reasonably well. The mean stoichiometric mixture fraction isosurface closes at approximately 15 nozzle diameters downstream from the injector, significantly upstream from the location where autoignition kernels appear. 
For the conditions considered and for the two turbulence intensity levels imposed at the coflow, the 'random spots' regime behaviour emerged in the simulations, in which AISs were generated continuously and advected downstream by the flow without establishing a strongly burning flame. The build up of the radical pool was initiated by the formation of $\mathrm{HO}_{2}$ at the local most reactive mixture fraction. The latter was found to vary significantly in the domain as a result of the strongly varying scalar dissipation rate, which was high close to the fuel injector, but decayed quickly to very low values farther downstream. It was observed that well-defined $\mathrm{HO}_{2}$ kernels were formed far enough from the injector, all of them eventually experiencing the thermal runaway at the AISs farther downstream.

The AISs appeared on the isosurface of the most reactive mixture fraction which can be computed a priori from homogeneous autoignition calculations; for the conditions considered here, the choice of the kinetic scheme was found to have a non-negligible effect on the computed $\xi_{M R}$ value and range of mixture fraction values corresponding to high reaction rates. Since at the range of heights where AISs appear the scalar dissipation rate is very low, the present study confirms the findings of previous numerical investigations in decaying homogeneous turbulence (i.e. in the absence of strong advection) with respect to the spot location in mixture fraction space. The AISs appeared at heights larger than 22 nozzle diameters, and therefore at conditions significantly leaner than stoichiometric, and resulted in short-lived PIFs, propagating edge flames which in some cases merged, or collided and annihilated each other. Deprived of fuel or air, PIFs cannot establish a strongly burning continuous flame, and are convected out of the domain by the flow. It is conceivable, however, that strong enough flow perturbations resulting in the appearance of AISs within the area enclosed by $\xi_{M R}$ can lead to lifted-flame stabilization, and thus to different combustion behaviours for the same nominal operating conditions.

The global qualitative features of the spots were in good agreement with the experiments, and the average frequency with which the spots appeared in the simulations as well as their spatial distribution matches well the measured values at similar conditions. Despite the relatively low number of AISs that could be afforded during the simulated time, a significant spread of their locations in physical space was observed, which can be accounted for by the flapping of the $\xi_{M R}$ isosurface.

Although a Lagrangian approach is the natural way to describe this case, it is not obvious what is the feature that should be tracked in time and space. Particles that at a certain time instant were located in high $\mathrm{HO}_{2}$ concentration kernels were tracked forward and backward in time, but their paths were found to differ significantly from the path of the kernels themselves. An attempt was also made to track the $\mathrm{HO}_{2}$ kernels, which forward in time were found to autoignite. However, these kernels could not be followed towards the injector where the high scalar dissipation rate is expected to have a significant effect as their concentration quickly decreased to very low values.

It is also not obvious how to translate the AIS locations into time so that different homogeneous and inhomogeneous autoignition set-ups can be compared. In an attempt to obtain a better estimate of a characteristic velocity than the bulk flow velocity to convert AIS locations to time, the combined effect of advection and drift due to diffusion of most reactive mixture 'pockets' through the domain was considered. The mean axial and radial locations and scatter of the autoignition kernels could be explained adequately by considering the propagation of these $\xi_{M R}$ isosurface elements.

In agreement with the experimental observations, the increased turbulence intensity resulted in the slightly delayed appearance of the first kernel, a downstream shift of autoignition and considerable scatter of the autoignition kernels. Interestingly, the 
former observation is not in agreement with three-dimensional DNSs of stagnant mixing layers (Sreedhara \& Lakshmisha 2002) where enhanced turbulence levels were shown to either have no effect on or to accelerate autoignition. The strong advection in the current set-up appears to have a significant effect on autoignition.

Future work will focus on the characterization of the combustion mode of the autoignition kernels using chemical explosive mode analysis ( $\mathrm{Lu}$ et al. 2010) as well as the structure and propagation characteristics of the PIFs. Simulations with different inflow turbulence intensities and length scales are also planned to study the turbulence-chemistry interactions in more detail and construct a database which can assist in the validation and development of transported p.d.f., flamelet and CMC models.

\section{Acknowledgements}

The authors would like to thank E. Mastorakos for his invaluable insight, H. Childs for his help with the visualizations using VisIt and R. Mathie for improving the figures. The simulations were performed at the Swiss National Supercomputing Center (CSCS). The financial support of the Swiss Federal Institute of Technology (ETH) under grant number TH-21-06-3 is gratefully acknowledged.

\section{REFERENCES}

del Álamo, G., Williams, F. A. \& SÁnchez, A. L. 2004 Hydrogen-oxygen induction times above crossover temperatures. Combust. Sci. Technol. 176 (10), 1599-1626.

Baritaud, T. A., Heinze, T. A. \& Coz, J. F. LE. 1994 Spray and self-ignition visualization in a DI Diesel engine. SAE Paper, 940681.

Bilger, R. W., StÅrner, S. H. \& KeE, R. J. 1990 On reduced mechanism for methane - air combustion in nonpremixed flames. Combust. Flame 80 (2), 135-149.

Blouch, J. D. \& LAW, C. K. 2003 Effects of turbulence on nonpremixed ignition in heated counterflow. Combust. Flame 132 (3), 512-522.

Blouch, J. D., Sung, C. J., Fotache, C. G. \& LAw, C. K. 1998 Turbulent ignition of non-premixed hydrogen by heated counterflowing atmospheric air. Proc. Combust. Inst. 27 (1), 1221-1228.

Cabra, R., Chen, J.-Y., Dibble, R. W., Karpetis, A. N. \& Barlow, R. S. 2005 Lifted methane-air jet flames in a vitiated coflow. Combust. Flame 143 (4), 491-506.

Cabra, R., Myhrvold, T., Chen, J. Y., Dibble, R. W., Karpetis, A. N. \& Barlow, R. S. 2002 Simultaneous laser Raman-Rayleigh-LIF measurements and numerical modeling results of a lifted turbulent $\mathrm{H}_{2} / \mathrm{N}_{2}$ jet flame in a vitiated coflow. Proc. Combust. Inst. 29 (2), 1881-1888.

Conaire, M. Ó. , Curran, H. J., Simmie, J. M., Pitz, W. J. \& Westbrook, C. K. 2004 A comprehensive modeling study of hydrogen oxidation. Intl J. Chem. Kinet. 36 (11), 603-622.

Dally, B. B., Karpetis, A. N. \& BARlow, R. S. 2002 Structure of turbulent nonpremixed jet flames in a diluted hot coflow. Proc. Combust. Inst. 29 (1), 1147-1154.

Deville, M. O., Fischer, P. F. \& Mund, E. H. 2002 High-order Methods for Incompressible Fluid Flows. Cambridge University Press.

ECHEKKI, T. \& CHEN, J. H. 2003 Direct numerical simulations of auto-ignition in nonhomogeneous hydrogen-air mixtures. Combust. Flame 134 (3), 169-191.

Fischer, P. F., Lottes, J. W. \& Kerkemeier, S. G. 2011 nek5000 web page http://nek5000.mcs. anl.gov.

GiBSON, C. H. 1968 Fine structure of scalar fields mixed by turbulence: I. Zero-gradient points and minimal gradient surfaces. Phys. Fluids 11 (11), 2305-2315. 
Gordon, R. L., Masri, A. R. \& Mastorakos, E. 2008 Simultaneous Rayleigh temperature, OHand $\mathrm{CH}_{2}$ O-LIF imaging of methane jets in a vitiated coflow. Combust. Flame 155 (1-2), $181-195$.

Hilbert, R. \& ThÈvenin, D. 2002 Autoignition of turbulent non-premixed flames investigated using direct numerical simulations. Combust. Flame 128 (1-2), 22-37.

Hindmarsh, A. C., Brown, P. N., Grant, K. E., Lee, S. L., Serban, R., Shumaker, D. E. \& WOODWARD, C. S. 2005 SUNDIALS: suite of nonlinear and differential/algebraic equation solvers. ACM Trans. Math. Softw. 31 (3), 363-396.

HuAng, M.-J. \& Leonard, A. 1994 Power-law decay of homogeneous turbulence at low Reynolds numbers. Phys. Fluids 6 (11), 3765-3775.

IM, H. G., Chen, J. H. \& LAW, C. K. 1998 Ignition of hydrogen-air mixing layer in turbulent flows. Proc. Combust. Inst. 27, 1047-1056.

Kerkemeier, S. G. 2010 Direct numerical simulation of combustion on petascale platforms: application to turbulent non-premixed hydrogen autoignition. PhD thesis, Swiss Federal Institute of Technology (ETH), Zurich, Switzerland.

KIM, I. S. 2004 Conditional moment closure for non-premixed turbulent combustion. PhD thesis, University of Cambridge, Cambridge, UK.

Klein, M., SAdiki, A. \& JAnickA, J. 2003 A digital filter based generation of inflow data for spatially developing direct numerical or large eddy simulations. J. Comput. Phys. 186 (2), 652-665.

LEe, Y. Y. \& Pope, S. B. 1995 Nonpremixed turbulent reacting flow near extinction. Combust. Flame 101 (4), 501-528.

Lemoine, F., Antoine, Y., Wolff, M. \& Lebouche, M. 2000 Some experimental investigations on the concentration variance and its dissipation rate in a grid generated turbulent flow. Intl $J$. Heat Mass Transfer. 43 (7), 1187-1199.

Li, J., Zhao, Z., KaZAKov, A. \& DrYer, F. L. 2004 An updated comprehensive kinetic model of hydrogen combustion. Intl J. Chem. Kinet. 36 (10), 566-575.

LiñÁn, A. \& CREspo, A. 1976 An asymptotic analysis of unsteady diffusion flames for large activation energies. Combust. Sci. Technol. 14 (1), 95-117.

Lu, T. F., Yoo, C. S., Chen, J. H. \& LAW, C. K. 2010 Three-dimensional direct numerical simulations of a turbulent lifted hydrogen jet flame in heated coflow: a chemical explosive mode analysis. J. Fluid Mech. 652, 45-64.

MARKIDES, C. N. 2005 Autoignition in turbulent flows. PhD thesis, University of Cambridge, Cambridge, UK.

MARKIDES, C. N. \& MASTORAKOS, E. 2005 An experimental study of hydrogen autoignition in a turbulent co-flow of heated air. Proc. Comb. Inst. 30 (1), 883-891.

MARKides, C. N. \& MAStorakos, E. 2006 Measurements of scalar dissipation in a turbulent plume with planar laser-induced fluorescence of acetone. Chem. Engng Sci. 61 (9), 2835-2842.

MARKides, C. N. \& MAStorakos, E. 2008a Flame propagation following the autoignition of axisymmetric hydrogen, acetylene and normal-heptane plumes in turbulent co-flows of hot air. J. Engng Gas Turbine Power 130, 011502.

Markides, C. N. \& Mastorakos, E. $2008 b$ Measurements of the statistical distribution of the scalar dissipation rate in turbulent axisymmetric plumes. Flow Turb. Combust. 81 (1-2), 221-234.

Markides, C. N. \& Mastorakos, E. 2011 Experimental investigation of the effects of turbulence and mixing on autoignition chemistry. Flow Turbul. Combust. 86 (3-4), 585-608.

Markides, C. N., De Paola, G. \& Mastorakos, E. 2007 Measurements and simulations of mixing and autoignition of an $n$-heptane plume in a turbulent flow of heated air. Exp. Thermal Fluid Sci. 31 (5), 393-401.

Mastorakos, E. 2009 Ignition of turbulent non-premixed flames. Prog. Energy Combust. Sci. 35 (1), 57-97.

Mastorakos, E., Baritaud, T. A. \& Poinsot, T. J. $1997 a$ Numerical simulations of autoignition in turbulent mixing flows. Combust. Flame 109 (1-3), 198-223. 
Mastorakos, E., Da Cruz, A. P., Baritaud, T. A. \& Poinsot, T. J. 1997b A model for the effects of mixing on the autoignition of turbulent flows. Comb. Sci. Technol. 125 (1-6), 243-282.

Medwell, P. R., Kalt, P. A. M. \& Dally, B. B. 2007 Simultaneous imaging of oh, formaldehyde, and temperature of turbulent nonpremixed jet flames in a heated and diluted coflow. Combust. Flame 148 (1-2), 48-61.

Medwell, P. R., Kalt, P. A. M. \& Dally, B. B. 2008 Imaging of diluted turbulent ethylene flames stabilised on a jet in hot coflow (JHC) burner. Combust. Flame 152 (1-2), 100-113.

Mizutani, Y., NAKABE, K. \& ChUnG, J. D. 1990 Effects of turbulent mixing on spray ignition. Proc. Combust. Inst. 23 (1), 1455-1460.

Mohamed, M. S. \& LARue, J. C. 1990 The decay power law in grid-generated turbulence. J. Fluid Mech. 219, 195-214.

Moin, P. \& MAHESh, K. 1998 Direct numerical simulation: a tool in turbulence research. Annu. Rev. Fluid Mech. 30, 539-578.

NAKAmura, I., SAKai, Y. \& MiYata, M. 1987 Diffusion of matter by a non-buoyant plume in grid-generated turbulence. J. Fluid Mech. 178, 379-403.

O'BRIEN, E. E. \& JiANG, T.-L. 1991 The conditional dissipation rate of an initially binary scalar in homogeneous turbulence. Phys. Fluids A 3 (12), 3121-3123.

Oldenhof, E., Tummers, M. J., van Veen, E. H. \& Roekaerts, D. J. E. M. 2010 Ignition kernel formation and lift-off behaviour of jet-in-hot-coflow flames. Combust. Flame 157 (6), $1167-1178$.

Peters, N. 1983 Local quenching due to flame stretch and non-premixed turbulent combustion. Combust. Sci. Technol. 30 (1-6), 1-17.

Pizza, G., Frouzakis, C. E., Mantzaras, J., Tomboulides, A. G. \& Boulouchos, K. 2010 Three-dimensional simulations of premixed hydrogen/air flames in microtubes. J. Fluid Mech. 658, 463-491.

Pope, S. B. 2000 Turbulent Flows. Cambridge University Press.

SReEdhara, S. \& LAKshmisha, K. N. 2000 Direct numerical simulation of autoignition in a nonpremixed, turbulent medium. Proc. Combust. Inst. 28 (1), 25-33.

SReedharA, S. \& LAKShmisha, K. N. 2002 Assessment of conditional moment closure models for turbulent autoignition using DNS data. Proc. Combust. Inst. 29 (2), 2051-2059.

Sutton, O. G. 1932 A theory of eddy diffusion in the atmosphere. Proc. R. Soc. Lond. A 135 (826), 143-165.

Tomboulides, A. G., LeE, J. C. Y. \& OrZAG, S. A. 1997 Numerical simulation of low Mach number reactive flows. J. Sci. Comput. 12, 139-167.

Viggiano, A. \& MAGi, V. 2004 A 2-D investigation of $n$-heptane autoignition by means of direct numerical simulation. Combust. Flame 137 (4), 432-443.

Wong, C. L. \& SteERE, D. E. 1982 The effects of diesel fuel properties and engine operating conditions on ignition delay. SAE Paper, 821231.

Wu, Z., MASRI, A. R. \& BILGER, R. W. 2006 An experimental investigation of the turbulence structure of a lifted $\mathrm{H}_{2} / \mathrm{N}_{2}$ jet flame in a vitiated co-flow. Flow Turbul. Combust. 76 (1), 61-81.

YetTer, R. A., DRYER, F. L. \& RABitZ, H. 1991 A comprehensive reaction mechanism for carbon monoxide/hydrogen/oxygen kinetics. Combust. Sci. Technol. 79 (1-3), 97-128.

Yoo, C. S., Richardson, E. S., Chen, R. \& SAnkaran, J. H. 2011 A DNS study on the stabilization mechanism of a turbulent lifted ethylene jet flame in highly-heated coflow. Proc. Combust. Inst. 33 (1), 1619-1627.

Yoo, C. S., SANKARAn, R. \& CHEN, J. H. 2009 Three-dimensional direct numerical simulation of a turbulent lifted hydrogen jet flame in heated coflow: flame stabilization and structure. J. Fluid Mech. 640, 453-481. 